Bull. Soc. math. France

132 (4), 2004, p. 509-541

\title{
INNER AND OUTER HAMILTONIAN CAPACITIES
}

\author{
BY DAVID HERMANN
}

\begin{abstract}
The aim of this paper is to compare two symplectic capacities in $\mathbb{C}^{n}$ related with periodic orbits of Hamiltonian systems: the Floer-Hofer capacity arising from symplectic homology, and the Viterbo capacity based on generating functions. It is shown here that the inner Floer-Hofer capacity is not larger than the Viterbo capacity and that they are equal for open sets with restricted contact type boundary. As an application, we prove that the Viterbo capacity of any compact Lagrangian submanifold is nonzero.

RÉSumÉ (Capacités hamiltoniennes intérieure et extérieure). - Nous nous proposons de comparer deux capacités dans $\mathbb{C}^{n}$ définies par les orbites périodiques de systèmes hamiltoniens. La première est la capacité de Floer-Hofer, issue de l'homologie symplectique; la seconde est la capacité de Viterbo basée sur des fonctions génératrices. Nous montrons que la capacité intérieure de Floer-Hofer n'est pas plus grande que celle de Viterbo et qu'elles coïncident sur les ouverts dont le bord est une variété de contact restreinte. Nous montrons enfin que la capacité de Viterbo d'une sous-variété lagrangienne compacte n'est jamais nulle.
\end{abstract}

\section{Contents}

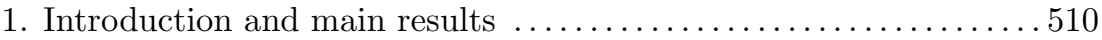

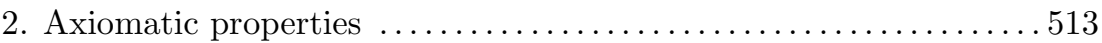

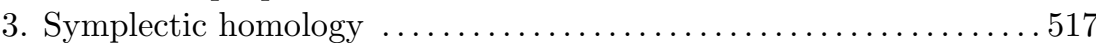

Texte reçu le $1^{\text {er }}$ février 2002, révisé le 10 mars 2003, accepté le 16 septembre 2003 David Hermann, Université de Paris 7, Institut de Mathématiques, Case 7012, 2 place Jussieu, 75251 Paris Cedex 05 (France) • E-mail : hermann@math.jussieu.fr 2000 Mathematics Subject Classification. - 53D40.

Key words and phrases. - Symplectic capacities, Lagrangian submanifolds. 


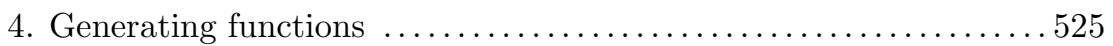

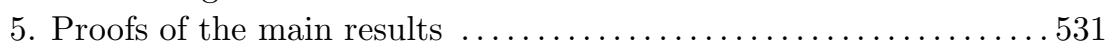

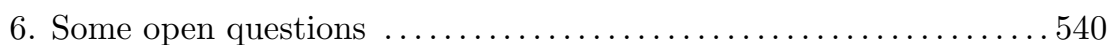

Bibliography ............................................... 540

\section{Introduction and main results}

Throughout this paper, we consider the symplectic space $\left(\mathbb{C}^{n}, \omega=\mathrm{d} \lambda_{0}\right)$, where $n \geqslant 2$ and $\lambda_{0}=\frac{1}{2} \operatorname{Im}(\bar{z} \cdot \mathrm{d} z)$. To each (time-dependent) Hamiltonian function $H \in \mathcal{H}_{\mathrm{t}}=C^{\infty}\left(S^{1} \times \mathbb{C}^{n}\right)$ we associate a Hamiltonian vector field $X_{H}$ given by $\omega\left(X_{H},.\right)=-\mathrm{d} H(t,$.$) . We shall always assume that X_{H}$ is complete: its flow $\phi_{t}^{H}$ is called the Hamiltonian flow of $H$. We denote the group of compactly supported Hamiltonian diffeomorphisms by $\mathcal{D}$ :

$$
\mathcal{D}=\left\{\phi_{1}^{H} / H \in C_{0}^{\infty}\left(S^{1} \times \mathbb{C}^{n}\right)\right\} .
$$

The symplectic size of subsets of $\mathbb{C}^{n}$ is measured by symplectic capacities introduced by Gromov in [6] and developed by Ekeland and Hofer in [3].

Definition 1.1. - A (relative) symplectic capacity on $\left(\mathbb{C}^{n}, \omega\right)$ is a map which associates a number $c(U) \in[0,+\infty]$ to each subset $U$ of $\mathbb{C}^{n}$ and which satisfies:

1) $U \subset V \Rightarrow c(U) \leqslant c(V)$ (monotonicity);

2) $c(\phi(U))=c(U)$ for any $\phi \in \mathcal{D}$ (symplectic invariance);

3) $c(\alpha U)=\alpha^{2} c(U)$ for any real number $\alpha>0$ (homogeneity);

4) $c\left(B^{2 n}(1)\right)=c\left(B^{2}(1) \times \mathbb{C}^{n-1}\right)=\pi$, where $B^{2 n}(1)$ is the unit open ball (normalization).

Given any capacity $c$, define the associated inner capacity $\stackrel{v}{c}$ and outer capacity $\hat{c}$ by

$$
\left\{\begin{array}{l}
\stackrel{\vee}{c}(U)=\sup \{c(K) / K \text { is compact and } K \subset U\}, \\
\hat{c}(U)=\inf \{c(V) / V \text { is open and } \bar{U} \subset V\}
\end{array}\right.
$$

The capacity $c$ is said to be inner regular if $\stackrel{v}{c}=c$ and outer regular if $\hat{c}=c$ (see [8]).

We will consider Hamiltonian capacities in $\mathbb{C}^{n}$, as introduced in [3]. Given any bounded connected open set $U \subset \mathbb{C}^{n}$, let $\mathcal{H}_{\text {ad }}(U) \subset \mathcal{H}_{\mathrm{t}}$ be some class of "admissible" Hamiltonian functions. Consider the action functional

$$
A_{H}(\gamma)=\int_{S^{1}} \gamma^{*} \lambda_{0}-\int_{0}^{1} H(t, \gamma(t)) \mathrm{d} t \quad \text { for } \gamma \in \Lambda=C^{\infty}\left(S^{1}, \mathbb{C}^{n}\right)
$$

TOME $132-2004-\mathrm{N}^{\mathrm{O}} 4$ 
whose critical points are the 1-periodic orbits of $X_{H}$. By a universal variational process, select a positive critical value $c(H)$ of $A_{H}$ for each $H \in \mathcal{H}_{\text {ad }}(U)$. Depending on the functorial properties of $\mathcal{H}_{\text {ad }}$ (see Section 2), define the capacity of $U$ by one of the following formulae

$$
\left\{\begin{array}{l}
c(U)=\sup \left\{c(H) / H \in \mathcal{H}_{\mathrm{ad}}(U)\right\} \quad \text { or } \\
c(U)=\inf \left\{c(H) / H \in \mathcal{H}_{\mathrm{ad}}(U)\right\} .
\end{array}\right.
$$

Then extend this capacity to all subsets of $\mathbb{C}^{n}$ by standard processes: the capacity of any open set $U$ is given by

$$
c(U)=\sup \{c(V) / V \text { open, bounded and connected with } V \subset U\}
$$

and the capacity of any subset $E$ in $\mathbb{C}^{n}$ is given by

$$
c(E)=\inf \{c(U) / U \text { is open and } E \subset U\} .
$$

These capacities have a geometric representation in the following situation.

DEFINITION 1.2. - A hypersurface $\Sigma$ has restricted contact type (or RCT) if there exists a vector field $\eta$ satisfying $\eta \pitchfork \Sigma$ and $L_{\eta} \omega=\omega$ on $\mathbb{C}^{n}$, where $L$ denotes the Lie derivative. A bounded connected open set with RCT boundary will be called a $R C T$ open set.

The vector field $\eta$ is called a Liouville vector field. Each Hamiltonian capacity satisfies the Representation Theorem: the capacity of any RCT open set $U$ is the area of some closed characteristic of $\partial U$.

We will focus here on two of these Hamiltonian capacities. The first one was first defined in [5] using symplectic homology (see [4]). This capacity can be viewed as a variant of the Ekeland-Hofer capacity in [3]. The admissible class $\mathcal{H}_{\mathrm{FH}}(U)$ is the set of those Hamiltonian functions which are negative near $S^{1} \times U$ and quadratic at infinity, and the critical value $c_{\mathrm{FH}}(H)$ is obtained by considering the Floer homology groups associated to $H$. We will also consider the generating function capacity defined by Viterbo in [14]. The admissible class $\mathcal{H}_{\mathrm{V}}(U)$ is the set of compactly supported Hamiltonian functions with support in $S^{1} \times U$, and the critical value $c_{\mathrm{V}}(H)$ is defined as a minmax critical value for a generating function of the graph of $\phi_{1}^{H}$. It should be noticed that the capacity $c$ in [14] is defined a priori for disconnected open sets. Thus the capacity $c_{\mathrm{V}}$ defined by (1.3) could be smaller than $c$ : if $U_{1}$ and $U_{2}$ are disjoint open sets, $(1.3)$ shows that $c_{\mathrm{V}}\left(U_{1} \cup U_{2}\right)=\max \left(c_{\mathrm{V}}\left(U_{1}\right), c_{\mathrm{V}}\left(U_{2}\right)\right)$, whereas this property is known for the capacity $c$ only if $U_{1}$ and $U_{2}$ can be separated by an hyperplane (see [12]). However, this does not affect the results in this paper. A simple observation proves the following regularity result.

Proposition 1.3. - For any subset $U$ in $\mathbb{C}^{n}$, we have

$$
c_{\mathrm{V}}(U)=\check{c}_{\mathrm{V}}(U) \text { and } \quad c_{\mathrm{FH}}(U)=\hat{c}_{\mathrm{FH}}(U) .
$$


Several properties of $c_{\mathrm{FH}}$ and $c_{\mathrm{V}}$ make the interest of comparing them. Because of Proposition 1.3, it is easy to find open sets $U$ with $c_{\mathrm{FH}}(U)=1$ and $c_{\mathrm{V}}(U)$ arbitrarily small. But this occurs only because the periodic orbits defining $c_{\mathrm{FH}}(U)$ stay away from $U$, whereas those defining $c_{\mathrm{V}}(U)$ lie in $U$. This phenomenon is somehow artificial and it disappears if we compare capacities with the same regularity: our main result is the following inequality.

TheOREM 1.4. - For any subset $U$ in $\mathbb{C}^{n}$, we have $\stackrel{\vee}{\mathrm{FH}}(U) \leqslant c_{\mathrm{V}}(U)$.

The main feature in Theorem 1.4 is that $\stackrel{v}{c}_{\mathrm{FH}}$ measures a set from inside, whereas $c_{\mathrm{V}}$ measures it from outside, which heuristically explains the inequality. Moreover, by the homogeneity property, any symplectic capacity $c$ satisfies

$$
\stackrel{\vee}{c}(U)=\hat{c}(U)=c(U) \text { for any RCT open set } U
$$

(see Section 2). This leads to $c_{\mathrm{FH}}(U) \leqslant c_{\mathrm{V}}(U)$, and we will also prove the opposite inequality.

TheOREM 1.5. - For any RCT open set $U$ in $\mathbb{C}^{n}$, we have $c_{\mathrm{FH}}(U)=c_{\mathrm{V}}(U)$.

Our main application of Theorem 1.4 deals with the so-called Lagrangian camel problem. Set

$$
\begin{gathered}
E_{-}=\left\{z \in \mathbb{C}^{n} / \operatorname{Re}\left(z_{1}\right)<0\right\}, \quad E_{+}=\left\{z \in \mathbb{C}^{n} / \operatorname{Re}\left(z_{1}\right)>0\right\}, \\
E(\varepsilon)=E_{-} \cup E_{+} \cup B^{2 n}(\varepsilon)
\end{gathered}
$$

and consider some compact set $L \subset E_{-}$. The camel problem is formulated as follows:

Does there exist $H \in \mathcal{H}_{t}$ with support in $S^{1} \times E(\varepsilon)$ satisfying $\phi_{1}^{H}(L) \subset E_{+}$?

By the symplectic reduction properties of the capacity $c_{V}$, a positive answer implies $c_{V}(L) \leqslant \pi \varepsilon^{2}$ (see [14]). In [10], Théret proved that compact hyperbolic Lagrangian submanifolds and Lagrangian tori have nonzero Viterbo capacity. In other terms, such a submanifold cannot pass through an arbitrarily small hole made in a hyperplane. On the other hand, we can deduce from results by Viterbo in [16] that the Floer-Hofer capacity of any compact Lagrangian submanifold $L$ in $\mathbb{C}^{n}$ is nonzero. More precisely, let $\mathcal{J}$ be the set of almost complex structures $J$ on $\mathbb{C}^{n}$ satisfying $J=i$ at infinity and calibrated by $\omega$, which means that $\omega(., J$.) is a Riemannian metric. For each $J \in \mathcal{J}$, consider the set $\mathcal{C}_{J}$ of $J$-holomorphic curves with boundary in $L$. The Gromov Compactness Theorem shows that

$$
\widetilde{w}(L)=\sup _{J \in \mathcal{J}}\left(\inf _{C \in \mathcal{C}_{J}} \int_{C} \omega\right)>0,
$$

and the following inequality holds (compare [16], Theorem 6.10).

THEOREM 1.6. - For any compact Lagrangian submanifold L, we have

$$
c_{F H}(L) \geqslant \widetilde{w}(L) .
$$

TOME $132-2004-\mathrm{N}^{\mathrm{O}} 4$ 
Since we have $\stackrel{\vee}{\mathrm{FH}}_{\mathrm{FH}}(L)=c_{\mathrm{FH}}(L)$ for any compact set $L$ (see Section 2), Theorems 1.4 and 1.6 imply the following generalization of [10].

COROLlary 1.7. - If $L$ is a compact Lagrangian submanifold, we have

$$
c_{\mathrm{V}}(L) \geqslant c_{\mathrm{FH}}(L)>0 .
$$

Let $\left[\lambda_{0}\right] \in H^{1}(L)$ be the Liouville class of $L$ and let $\mathcal{P}(L)=\left[\lambda_{0}\right] \cdot \pi_{1}(L)$ be its periods group: by the Gromov Compactness Theorem, we have $\widetilde{w}(L) \in \mathcal{P}(L)$. When $L$ is rational, that is, $\mathcal{P}(L)=a \mathbb{Z}$ for some real number $a>0$, Theorem 1.6 implies that $L$ cannot be moved by a Hamiltonian isotopy into an open set with capacity smaller than $a$, and Theorem 1.7 implies that $L$ cannot pass through a hole of radius less than $\sqrt{a / \pi}$.

In Section 2 we will recall the common features of Hamiltonian capacities, and establish some very elementary results about them, in particular Proposition 1.3 and (1.5). In Section 3 we recall the definitions of symplectic homology and of the Floer-Hofer capacity in [4], [1], [5], [16], [7]. In Section 4 we recall the definition of the Viterbo capacity in [14] and the uniqueness of symplectic homology proved in [15], which is the main tool in the proof of Theorem 1.4. We also give our strategy, explaining how $c_{\mathrm{V}}$ and $\stackrel{\vee}{c}_{\mathrm{FH}}$ can be viewed as differences of critical levels of the same Hamiltonian function. In Section 5 we prove Theorems 1.4, 1.5 and 1.6. The proof of Theorem 1.5 involves the intrinsic description of the capacity $c_{\mathrm{FH}}$ given in [16] and [7], followed by a deformation argument. In the proof of Theorem 1.6, we adapt the arguments in [16] to our settings.

Acknowledgments. - I wish to thank Claude Viterbo for many helpful discussions, and the participants at the Geometry and Analysis seminar in Jussieu, especially Marc Chaperon, for their patient audience of my "marathon talks" on these subjects. Special thanks to Laurent Lazzarini for his help in correcting this paper.

\section{Axiomatic properties}

Consider some functor $\mathcal{H}_{\text {ad }}$ which associates a class of Hamiltonian functions $\mathcal{H}_{\text {ad }}(U) \subset \mathcal{H}_{\mathrm{t}}$ to each bounded connected open set $U$ in $\mathbb{C}^{n}$. Set

$$
\mathcal{H}_{\mathrm{ad}}=\bigcup_{U \subset \mathbb{C}^{n}} \mathcal{H}_{\mathrm{ad}}(U)
$$

and consider a positive section $c$ of the action spectrum, that is, a map $c$ : $\mathcal{H}_{\text {ad }} \rightarrow \mathbb{R}$ such that $c(H)=A_{H}\left(\gamma_{H}\right)$ is a positive critical value of $A_{H}$. Assume that the selector $c$ is invariant by Hamiltonian isotopies, which means that $H \circ \phi \in \mathcal{H}_{\text {ad }}$ and $c(H \circ \phi)=c(H)$ for each $H \in \mathcal{H}_{\text {ad }}$ and each $\phi \in \mathcal{D}$. Given 
any real number $r>0$ and any Hamiltonian function $H \in \mathcal{H}_{\mathrm{t}}$, define

$$
\left(r^{2} \star H\right)(t, z)=r^{2} H\left(t, r^{-1} z\right),
$$

and assume that $c$ is homogeneous, which means that

$$
r^{2} \star H \in \mathcal{H}_{\mathrm{ad}} \quad \text { and } \quad c\left(r^{2} \star H\right)=r^{2} c(H)
$$

for each $H \in \mathcal{H}_{\text {ad }}$ and each $r>0$. Assume additionally that the functor $\mathcal{H}_{\text {ad }}$ is monotone with respect to inclusion, which means that $U \subset V$ implies either

$$
\mathcal{H}_{\text {ad }}(U) \subset \mathcal{H}_{\text {ad }}(V) \text { or } \quad \mathcal{H}_{\text {ad }}(V) \subset \mathcal{H}_{\text {ad }}(U) .
$$

Define the capacity of $U$ by

$$
c(U)=\left\{\begin{array}{l}
\sup \left\{c(H) / H \in \mathcal{H}_{\mathrm{ad}}(U)\right\} \text { in the first case, } \\
\inf \left\{c(H) / H \in \mathcal{H}_{\mathrm{ad}}(U)\right\} \text { in the last case. }
\end{array}\right.
$$

Obviously, our above assumptions on the selector $c$ imply the symplectic invariance, the homogeneity, and the monotonicity of the capacity $c$. In order to get the normalization, we should compute the capacity of ellipsoids. This can be done when the selector $c$ is monotone with respect to some partial ordering $\prec$ which makes $\mathcal{H}_{\text {ad }}(U)$ a directed set. A cofinal family in $\mathcal{H}_{\text {ad }}(U)$ is an increasing 1-parameter family $H_{\lambda} \in \mathcal{H}_{\text {ad }}(U)$ satisfying

$$
\forall H \in \mathcal{H}_{\mathrm{ad}}(U), \exists A \in \mathbb{R} \text { such that } \lambda>A \Rightarrow H \prec H_{\lambda},
$$

and for such a family, we have $c(U)=\lim _{\lambda \rightarrow+\infty} c\left(H_{\lambda}\right)$.

If $H_{1} \leqslant H_{2}$ on $S^{1} \times \mathbb{C}^{n}$, we have $A_{H_{1}} \geqslant A_{H_{2}}$ on $\Lambda$. Since the selector $c$ is obtained by a universal variational process, it is monotone with respect to the partial orderings $\prec$ and $\succ$ given by

$$
H_{1} \prec H_{2} \Longleftrightarrow H_{2} \succ H_{1} \Longleftrightarrow H_{1} \leqslant H_{2} \text { on } S^{1} \times \mathbb{C}^{n} .
$$

At this stage, we can distinguish two cases which fit into this general framework.

Case (CS). - Let $\mathcal{H}_{\mathrm{CS}}$ be the class of compactly supported Hamiltonian functions and set

$$
\mathcal{H}_{\mathrm{CS}}(U)=\left\{H \in \mathcal{H}_{t} \text { such that } \operatorname{Supp}(H) \subset S^{1} \times U\right\} .
$$

In this case, $U \subset V$ implies $\mathcal{H}_{\mathrm{CS}}(U) \subset \mathcal{H}_{\mathrm{CS}}(V)$ and we define

$$
c_{\mathrm{CS}}(U)=\sup \left\{c(H) / H \in \mathcal{H}_{\mathrm{ad}}(U)\right\} .
$$

The selector $c$ is increasing with respect $\succ$, and the "cofinal Hamiltonians" are very negative inside $U$ :

$$
\forall H \in \mathcal{H}_{\mathrm{CS}}(U), \exists A \in \mathbb{R} \text { such that } \lambda>A \Rightarrow H_{\lambda} \leqslant H \text { on } S^{1} \times \mathbb{C}^{n} .
$$



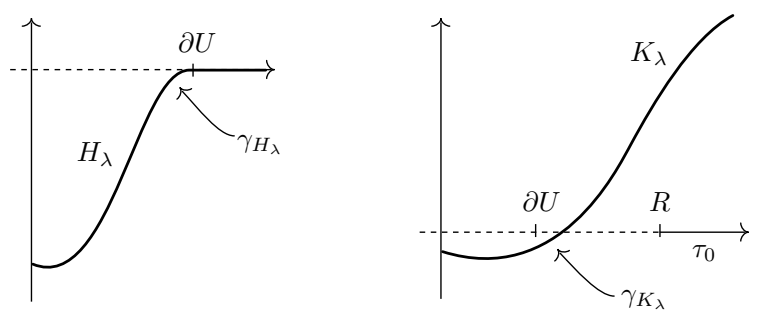

Figure 1. Cofinal families $H_{\lambda}$ in case (CS), $K_{\lambda}$ in case (QI)

Case (QI). - Let $\mathcal{H}_{\mathrm{QI}}$ be the class of those Hamiltonian function which are quadratic at infinity and somewhere negative and set

$$
\mathcal{H}_{\mathrm{QI}}(U)=\left\{\begin{array}{l}
H \in \mathcal{H}_{t} \text { such that } H(t, z)<0 \text { for any }(t, z) \in S^{1} \times \bar{U} \text { and } \\
H(t, z)=\mu \cdot \tau_{0}(z)+c \text { off a compact set for some } \mu, c \in \mathbb{R}
\end{array}\right\},
$$

where $\tau_{0}\left(z_{1}, \ldots, z_{n}\right)=\left|z_{1}\right|^{2}+\cdots+\left|z_{n}\right|^{2}$. Here, $U \subset V$ implies $\mathcal{H}_{\mathrm{QI}}(V) \subset \mathcal{H}_{\mathrm{QI}}(U)$ and we define

$$
c_{\mathrm{QI}}(U)=\inf \left\{c(H) / H \in \mathcal{H}_{\mathrm{ad}}(U)\right\} .
$$

The selector $c$ is decreasing with respect to $\prec$ and the "cofinal Hamiltonians" $K_{\lambda}$ are very small in $U$ and very large off $\bar{U}$ (see Figure 1 ):

$$
\forall K \in \mathcal{H}_{\mathrm{QI}}(U), \exists A \in \mathbb{R} \text { such that } \lambda>A \Rightarrow K_{\lambda} \geqslant K \text { on } S^{1} \times \mathbb{C}^{n} .
$$

Now consider the case of the unit open ball $B=B^{2 n}(1)$. We take Hamiltonian functions of the form $H=h \circ \tau_{0}$. Their action spectrum is given by

$$
A_{H}=s h^{\prime}(s)-h(s)
$$

where $h^{\prime}(s) \in \pi \mathbb{Z}$ (see Section 3.1).

- In case (QI), we perturb the ideal function given by

$$
h(s)= \begin{cases}0 & \text { if } s<1, \\ h(s)=\mu(s-1) & \text { if } s \geqslant 1\end{cases}
$$

(compare Figure 1), whose ideal action spectrum is $\pi \mathbb{Z} \cap] 0, \mu]$. The additional computation of the Conley-Zehnder index of the critical orbits shows that the capacity of the unit ball equals $\pi$.

- Similarly, in case (CS), we perturb the ideal function given by

$$
h(s)= \begin{cases}\lambda(s-1) & \text { if } s<1, \\ 0 & \text { if } s \geqslant 1,\end{cases}
$$

and this leads to the same conclusion and explains our choices of signs. Similar methods allow to compute the capacity of any ellipsoid, implying the normalization. 
The regularity of Hamiltonian capacities is a direct consequence of their definitions. Indeed, for any bounded connected open set $U$, we have

$$
\mathcal{H}_{\mathrm{CS}}(U)=\bigcup_{\bar{V} \subset U} \mathcal{H}_{\mathrm{CS}}(V) \text {, and } \mathcal{H}_{\mathrm{QI}}(U)=\bigcap_{\bar{U} \subset V} \mathcal{H}_{\mathrm{CS}}(V),
$$

which implies

$$
\begin{array}{ll}
c_{\mathrm{CS}}(U)=\sup \left\{c_{\mathrm{CS}}(V) / \bar{V} \subset U\right\}=\check{c}_{\mathrm{CS}}(U) & \text { in case (CS), } \\
c_{\mathrm{QI}}(U)=\inf \left\{c_{\mathrm{QI}}(V) / \bar{U} \subset V\right\}=\hat{c}_{\mathrm{QI}}(U) & \text { in case (QI). }
\end{array}
$$

This proves Proposition 1.3, since the capacity $c_{\mathrm{FH}}$ fits into Case (QI) and the capacity $c_{\mathrm{V}}$ fits into Case (CS) (see Sections 3.2 and 4.1). This difference of regularity shows that the capacities $c_{\mathrm{FH}}$ and $c_{\mathrm{V}}$ cannot be equal, even not equivalent. Indeed, let

$$
\mathbb{T}=\left\{\left(z_{1}, \ldots, z_{n}\right) \in \mathbb{C}^{n} /\left|z_{k}\right|=1,1 \leqslant k \leqslant n\right\}
$$

be the standard Lagrangian torus: we have (see Section 5.3)

$$
c_{\mathrm{V}}(\mathbb{T})=c_{\mathrm{FH}}(\mathbb{T})=\pi .
$$

For every small real number $\varepsilon>0$, let $U_{\varepsilon}$ be the following tubular neighborhood of $\mathbb{T}$

$$
U_{\varepsilon}=\left\{\left(z_{1}, \ldots, z_{n}\right) \in \mathbb{C}^{n} /\left.|| z_{k}\right|^{2}-1 \mid<\varepsilon, 1 \leqslant k \leqslant n\right\} .
$$

Make some cut in $U_{\varepsilon}$ by considering the open set

$$
V_{\varepsilon}=\left\{z \in U / \arg \left(z_{1}\right) \neq 0\right\} \text {. }
$$

Since the capacity $c_{\mathrm{FH}}$ is outer regular, we have

$$
c_{\mathrm{FH}}\left(V_{\varepsilon}\right)=c_{\mathrm{FH}}\left(U_{\varepsilon}\right) \geqslant \pi \text {. }
$$

On the other hand, we have

$$
V_{\varepsilon}=\bigcup_{\delta>0} V_{\varepsilon}^{\delta}
$$

where $V_{\varepsilon}^{\delta}=\left\{z \in \mathbb{C}^{n} /\left|\arg \left(z_{1}\right)\right|>\delta\right.$ and $\left.\left.|| z_{k}\right|^{2}-1 \mid<\varepsilon, 1 \leqslant k \leqslant n\right\}$. Using polar coordinates, it is easy to find a Hamiltonian isotopy $\phi \in \mathcal{D}$ such that

$$
\phi\left(V_{\varepsilon}^{\delta}\right) \subset B^{2}(\sqrt{2 \varepsilon}) \times \mathbb{C}^{n-1} .
$$

Since $c_{\mathrm{V}}$ is inner regular, we get

$$
c_{\mathrm{V}}\left(V_{\varepsilon}\right)=\sup \left\{c_{\mathrm{V}}\left(V_{\varepsilon}^{\delta}\right) / \delta>0\right\} \leqslant 2 \pi \varepsilon
$$

We have thus obtained a family of open sets $V_{\varepsilon}$ such that $c_{\mathrm{V}}\left(V_{\varepsilon}\right)$ is arbitrarily small and $c_{\mathrm{FH}}\left(V_{\varepsilon}\right) \geqslant \pi$. Heuristically, this is due to the fact that the periodic orbits with positive action of a cofinal family in $\mathcal{H}_{\mathrm{V}}(U)$ lie in $U$, whereas those of a cofinal family in $\mathcal{H}_{\mathrm{FH}}(U)$ stay away from $U$, as pointed out on Figure 1. This somehow artificial phenomenon disappears if we replace $c_{\mathrm{FH}}$ by $\breve{c}_{\mathrm{FH}}$ : in this case, we can choose cofinal families for each capacity so that all the relevant orbits lie in $U$. Notice that this difference of regularity disappears if we consider

TOME $132-2004-\mathrm{N}^{\mathrm{O}} 4$ 
compact sets, because (1.1) and (1.4) imply that any Hamiltonian capacity $c$ fulfills the condition

$$
\check{c}(L)=\hat{c}(L)=c(L) \text { for any compact subset } L \text { in } \mathbb{C}^{n} .
$$

Similarly, consider any RCT open set $U \subset \mathbb{C}^{n}$, and choose a Liouville vector field $\eta$ transversal to $\partial U$. By definition, its flow $\psi_{t}$ satisfies $\psi_{t}^{*} \omega=\mathrm{e}^{t} \omega$ on $\mathbb{C}^{n}$ for every $t \in \mathbb{R}$, and by homogeneity any symplectic capacity $c$ satisfies

$$
c\left(\psi_{t}(U)\right)=\mathrm{e}^{t} c(U) \text { for every } t \in \mathbb{R} .
$$

This implies some continuity property of $c$ near the boundary of $U$, namely that we have

$$
\check{c}(U)=c(U)=\hat{c}(U) .
$$

Moreover, by the monotonicity properties of the selector $c$, we can choose suitable cofinal families in $\mathcal{H}_{\mathrm{ad}}(U)$ in order to prove the Representation Theorem (see Section 5.2).

\section{Symplectic homology}

Here we recall the definition of the Floer-Hofer capacity (see [5]). We assume the reader to be familiar with symplectic homology (see [4], [1], [16]), and we just fix some notations: we point out that our conventions lead to a relative homology as in [4], whereas those in [16] lead to a cohomology.

3.1. Definitions and functorial properties. - Let $(M, \omega)$ be a connected compact symplectic manifold with RCT boundary $\Sigma=\partial M$. Let $c_{1}$ be the first Chern class of $T M$ endowed with an almost complex structure $J$ calibrated by $\omega$ : we assume that $c_{1}$ vanishes on spherical classes. Let $\eta$ be a Liouville vector field transversal to $\partial M$. The 1 -form $\lambda=i_{\eta} \omega=\omega(\eta,$.$) satisfies \mathrm{d} \lambda=\omega$, thus $M$ is exact, and its restriction $\alpha$ to $\Sigma$ is a contact form, which means that $\alpha \wedge(\mathrm{d} \alpha)^{n-1}$ is a volume form. In particular, the restriction of $\mathrm{d} \alpha$ to the contact field $\operatorname{Ker}(\alpha)$ is a symplectic form. The Reeb vector field associated to $\alpha$ is the unique vector field $X_{\alpha}$ on $\Sigma$ such that $i_{X_{\alpha}} \mathrm{d} \alpha=0$ and $\alpha\left(X_{\alpha}\right)=1$. The closed characteristics of $\Sigma$ are the periodic orbits of $X_{\alpha}$, and the action spectrum $\mathcal{S}(\Sigma)$ is the set of their periods. The symplectization of $\Sigma$ is the symplectic manifold

$$
(\widehat{\Sigma}=] 0,+\infty[\times \Sigma, d \widehat{\lambda}), \text { where } \widehat{\lambda}=\tau \cdot \pi^{*} \alpha
$$

and $\tau, \pi$ are the projections onto the factors. Observe that we have a symplectic splitting

$$
T \widehat{\Sigma}=\operatorname{Ker}(\alpha) \oplus\left(\mathbb{R} \widehat{\eta} \oplus \mathbb{R} X_{\alpha}\right) \text {, where } \widehat{\eta}(s, x)=s \partial / \partial s \text { fulfills } i_{\widehat{\eta}} \mathrm{d} \widehat{\lambda}=\widehat{\lambda} .
$$

Choose an almost complex structure $J_{0}$ calibrated by $\mathrm{d} \alpha$ on the symplectic vector bundle $(\operatorname{Ker}(\alpha), \mathrm{d} \alpha) \rightarrow \Sigma$, and extend it to $T \widehat{\Sigma}$ by $J_{0} \cdot \widehat{\eta}=X_{\alpha}$ and $J_{0} \cdot X_{\alpha}=-\widehat{\eta}$. The almost complex structure $J_{0}$ is calibrated by $\mathrm{d} \widehat{\lambda}$, and the 
function $\tau$ is plurisubharmonic with respect to $J_{0}$. Let $\psi_{t}$ be the flow of $\eta$, and observe that the formula

$$
\Psi\left(\mathrm{e}^{t}, x\right)=\psi_{t}(x)
$$

defines an embedding of the manifold ]0,1] $\times \Sigma$ into $M$ satisfying $\Psi^{*} \lambda=\widehat{\lambda}$, thus we obtain an exact symplectic manifold by setting

$$
(\widehat{M}, \widehat{\omega})=(M, \omega=\mathrm{d} \lambda) \bigcup_{\partial M=\Psi(\{1\} \times \Sigma)}([1,+\infty[\times \Sigma, \mathrm{d} \widehat{\lambda}) .
$$

Since we have $\Psi^{*} \eta=\widehat{\eta}$, the flow $\psi_{t}$ extends to $\widehat{M}$ and $\partial M$ has $\operatorname{RCT}$ in $\widehat{M}$. For any real number $\rho>0$, set $M^{\rho}=\psi_{\ln (\rho)}(M)$ : we have $\partial M^{\rho}=\tau^{-1}(\rho)$. Observe that the Hamiltonian vector field of the function $\tau$ on $\widehat{\Sigma}$ is given by $X_{\tau}=X_{\alpha}$. Consequently, if $h$ is a smooth function, the 1-periodic orbits of the autonomous Hamiltonian function $H=h \circ \tau$ on $\widehat{\Sigma}$ are closed characteristics of $\{s\} \times \Sigma$ with period $\left|h^{\prime}(s)\right|$ and action

$$
A_{H}(\gamma)=\int_{S^{1}} \gamma^{*} \lambda-\int_{0}^{1} H(t, \gamma(t)) \mathrm{d} t=s h^{\prime}(s)-h(s) \text {. }
$$

Definition 3.1. - Let $\mathcal{H}_{\mathrm{FH}}$ be the class of those smooth functions $H$ in $C^{\infty}\left(S^{1} \times \widehat{M}\right)$ such that

- $H=\mu \tau+c$ off a compact set, where $c, \mu \in \mathbb{R}$ fulfill $\mu \geqslant 0$ and $\mu \notin \mathcal{S}(\Sigma)$;

- there exists some nonempty open set $U \subset M$ such that $H<0$ on $S^{1} \times \bar{U}$;

- all 1-periodic orbits of $H$ are nondegenerate.

Given $H \in \mathcal{H}_{\mathrm{FH}}$, observe that all 1-periodic orbits of $H$ stay in a compact set. Let $i_{\mathrm{CZ}}$ denote the Conley-Zehnder index, normalized for contractible orbits by the formula

$$
i_{\mathrm{CZ}}(H ; x)=n-i_{\mathrm{M}}(H ; x),
$$

where $H$ is any $C^{2}$-small autonomous Hamiltonian function, $x$ is any nondegenerate critical point of $H$, and $i_{\mathrm{M}}$ denotes the Morse index (see [9]). For noncontractible orbits, some additional normalizing data must be chosen, see [2]. On the other hand, let $\mathcal{J}_{t}$ be the space of time-dependent almost complex structures $J$ on $\widehat{M}$ calibrated by $\widehat{\omega}$ such that $J(t,)=.J_{0}$ off a compact set (the time-dependence of $J$ is needed here for transversality reasons). The gradient lines of $A_{H}$ for the metric induced by $J$ are solutions of

$$
\frac{\partial u}{\partial s}+J(t, u) \frac{\partial u}{\partial t}=J(t, u) X_{H}(t, u) \text { for } u \in C^{\infty}\left(\mathbb{R} \times S^{1}, \widehat{M}\right)
$$

and are called Floer trajectories. Moreover, for a generic $J \in \mathcal{J}_{t}$ and for any 1-periodic orbits $x_{-}$and $x_{+}$of $H$, the set of Floer trajectories such that

$$
\lim _{s \rightarrow-\infty} u(s, .)=x_{-} \text {and } \lim _{s \rightarrow+\infty} u(s, .)=x_{+}
$$

TOME $132-2004-\mathrm{N}^{\mathrm{O}} 4$ 
is a manifold of dimension $i_{\mathrm{CZ}}\left(H ; x_{+}\right)-i_{\mathrm{CZ}}\left(H ; x_{-}\right)$endowed with a free $\mathbb{R}$-action $u \mapsto u\left(.+s_{0},.\right)$, and we call $\mathcal{M}\left(x_{-}, x_{+}\right)$the quotient manifold. Remember that the difference of action between the ends of a Floer trajectory is its energy:

$$
E_{J}(u)=\int_{\mathbb{R} \times S^{1}}\left|\frac{\partial u}{\partial s}\right|_{J}^{2} \mathrm{~d} t \mathrm{~d} s=A_{H}\left(x_{+}\right)-A_{H}\left(x_{-}\right),
$$

where $|\cdot|_{J}$ is the metric associated to $J$. Observe that, in any region where the Hamiltonian function $H$ is constant and the almost complex structure $J$ is time-independent (which will be allowed later on), any Floer trajectory is a $J$ holomorphic curve, and its energy equals its symplectic area. In [7] we proved the existence of a constant $C\left(J_{0}\right)>0$ such that if $H$ is constant and $J=J_{0}$ in $M^{B} \backslash M^{A}$, we have

(3.3) $E_{J}(u) \geqslant(B-A) C\left(J_{0}\right)$ for any Floer trajectory $u$ crossing $M^{B} \backslash M^{A}$.

Remember from [16] that the Floer trajectories for the Hamiltonian function $H=h \circ \tau$ and the almost complex structure $J_{0}$ satisfy the maximum principle, which means that the function $\tau \circ u$ has no local maximum. This shows that all trajectories which connect orbits of $H \in \mathcal{H}_{\mathrm{FH}}$ stay in a compact set. Since $\widehat{M}$ contains no holomorphic sphere, the non-compactness of the manifolds $\mathcal{M}\left(x_{-}, x_{+}\right)$is only due to the breaking of trajectories. In particular, if $\mathcal{M}\left(x_{-}, x_{+}\right)$is 0 -dimensional, it is compact, and we denote its cardinal modulo 2 by $\delta\left(x_{-}, x_{+}\right)$. Given any integer $k$ and any number $a \in \mathbb{R} \cup\{+\infty\}$, let $\mathcal{P}_{k}^{a}$ be the set of 1-periodic orbits $\gamma$ of $H$ such that $A_{H}(\gamma)<a$ and $i_{\mathrm{CZ}}(H ; \gamma)=k$, and consider the $\mathbb{Z}_{2}$-vector space they span

$$
C_{k}^{a}(H)=\bigoplus_{x \in \mathcal{P}_{k}^{a}} \mathbb{Z}_{2} \cdot x
$$

For any numbers $a, b \in \mathbb{R} \cup\{+\infty\}$ such that $a \leqslant b$, consider the quotient space

$$
C_{k}^{[a, b[}(H)=C_{k}^{b}(H) / C_{k}^{a}(H) .
$$

Define the boundary operator

$$
\partial_{k}^{[a, b[}: C_{k}^{[a, b[} \longrightarrow C_{k-1}^{[a, b[} \quad \text { by } \quad \partial_{k} x=\sum_{y \in C_{k-1}^{a}(H)} \delta(y, x) \cdot y \text { for } x \in C_{k}^{a}(H) .
$$

We have $\partial_{k}^{[a, b[} \circ \partial_{k+1}^{[a, b[}=0$, and the Floer homology groups associated to $H$ are given by

$$
S_{k}^{[a, b[}(H)=\operatorname{Ker} \partial_{k}^{[a, b[} / \operatorname{Im} \partial_{k+1}^{[a, b[}
$$

and are independent of $J$. Notice that in order to define these groups, we only need that all 1-periodic orbits of $H$ with action in the interval $[a, b[$ are nondegenerate. Moreover, in order to get a regular almost complex structure, it is enough to perturb a given $J \in \mathcal{J}_{t}$ in in an open set containing these orbits, since all the relevant trajectories must cross such an open set (see [7]). This way we can define the Floer homology of pairs $(H, J)$ satisfying (3.3). Given 
two Hamiltonian functions $H_{-}, H_{+} \in \mathcal{H}_{\mathrm{FH}}$ such that $H_{-} \leqslant H_{+}$on $S^{1} \times \widehat{M}$, the monotonicity morphism

$$
m\left(H_{-}, H_{+}\right): S_{k}^{[a, b[}\left(H_{-}\right) \longrightarrow S_{k}^{[a, b[}\left(H_{+}\right)
$$

is obtained as before by counting the number of solutions of the equation

$$
\frac{\partial u}{\partial s}+J_{s}(t, u) \frac{\partial u}{\partial t}=J_{s}(t, u) X_{H_{s}}(t, u) \text { for } u \in C^{\infty}\left(\mathbb{R} \times S^{1}, \widehat{M}\right),
$$

where $\left(H_{s}, J_{s}\right)$ is a monotone homotopy connecting $\left(H_{+}, J_{+}\right)$to $\left(H_{-}, J_{-}\right)$. The above properties of Floer trajectories also apply to these monotonicity trajectories, except that the maximum principle has to be refined (see [1]). This way we get a directed system: if $U \subset \widehat{M}$ is a relatively compact connected open set, define

$$
S_{k}^{[a, b[}(\widehat{M}, U)=\lim _{k} S_{k}^{[a, b[}(H),
$$

where the direct limit includes all Hamiltonian functions $H$ in the class

$$
\mathcal{H}_{\mathrm{FH}}(U)=\left\{H \in \mathcal{H}_{\mathrm{FH}} / H<0 \text { on } S^{1} \times \bar{U}\right\} .
$$

Let us recall some of the functorial properties of symplectic homology. If $U \subset V$, the inclusion $\mathcal{H}_{\mathrm{FH}}(V) \subset \mathcal{H}_{\mathrm{FH}}(U)$ and the monotonicity morphisms in $\mathcal{H}_{\mathrm{FH}}(U)$ define an inclusion morphism

$$
i_{U}^{*}: S_{k}^{[a, b[}(\widehat{M}, V) \longrightarrow S_{k}^{[a, b[}(\widehat{M}, U)
$$

which behaves functorially. Next, given any numbers $a, b, a^{\prime}, b^{\prime}$ such that $a \leqslant a^{\prime}$ and $b \leqslant b^{\prime}$, we have a map $C_{k}^{[a, b[}(H) \rightarrow C_{k}^{\left[a^{\prime}, b^{\prime}[\right.}(H)$ given by inclusions, which defines a natural map

$$
S_{k}^{[a, b[}(\widehat{M}, U) \longrightarrow S_{k}^{\left[a^{\prime}, b^{\prime}\right.}[(\widehat{M}, U) .
$$

Given any numbers $a, b, c$ such that $-\infty<a \leqslant b \leqslant c \leqslant+\infty$, we have an exact sequence

$$
0 \rightarrow C_{k}^{[a, b[}(H) \longrightarrow C_{k}^{[a, c[}(H) \longrightarrow C_{k}^{[b, c[}(H) \rightarrow 0
$$

which gives rise to an exact triangle

$$
S_{k}^{[a, b[}(\widehat{M}, U) \longrightarrow S_{k}^{[a, c[}(\widehat{M}, U) \longrightarrow S_{k}^{[b, c[}(\widehat{M}, U) \longrightarrow S_{k-1}^{[a, b[}(\widehat{M}, U) .
$$

This exact triangle is denoted by $\Delta^{a, b, c}(U)$ : it commutes with the inclusion morphisms. On the other hand, given any compactly supported Hamiltonian isotopy $\phi$ on $\widehat{M}$, the pullback $H \mapsto H \circ \phi$ defines an isomorphism

$$
\phi_{\#}: S_{k}^{[a, b[}(\widehat{M}, \phi(U)) \stackrel{\sim}{\longrightarrow} S_{k}^{[a, b[}(\widehat{M}, U) .
$$

If $\phi(U) \subset V$, the composition of $\phi_{\#}$ with the inclusion morphism defines a pullback morphism

$$
\phi^{*}: S_{k}^{[a, b[}(\widehat{M}, V) \longrightarrow S_{k}^{[a, b[}(\widehat{M}, U)
$$

TOME $132-2004-\mathrm{N}^{\mathrm{O}} 4$ 
which is compatible with all previous arrows. The Isotopy Invariance Theorem (see [4]) implies that if $\phi_{t}(U) \subset V$ for all $t \in[0,1]$, then we have $\phi_{1}^{*}=\phi_{0}^{*}$. Since the flow $\psi_{s}$ of $\eta$ fulfills $\psi_{s}^{*} \omega=\mathrm{e}^{s} \omega$, the computation of the Floer homology of $\mathrm{e}^{s} H\left(t, \psi_{-s}(z)\right)$ shows that

$$
S_{k}^{\left[e^{s} a, e^{s} b[\right.}\left(\widehat{M}, \psi_{s}(U)\right)=S_{k}^{[a, b[}(\widehat{M}, U) \quad \text { for any } s \in \mathbb{R} .
$$

Define the intrinsic symplectic homology groups of $M$ by

$$
I S_{k}^{[a, b[}(M)=S_{k}^{[a, b[}(\widehat{M}, M) .
$$

In order to compute these groups, we can use a natural cofinal family $K_{\lambda}$ in $\mathcal{H}_{\mathrm{FH}}(M)$ defined as follows. Choose real numbers $\delta>0$ and $\lambda$ such that $\delta$ is small enough and $\lambda \notin \mathcal{S}(\Sigma)$, and set $K_{\lambda}=-\delta$ in $M$ and $K_{\lambda}=-\delta+\lambda(\tau-1)$ off $M$. Smooth the corner and perturb $K_{\lambda}$ in $M$, so that $K_{\lambda}=f$ in $M$, where $f$ is some $C^{2}$-small Morse function having a unique local minimum $x_{0}$ satisfying $f\left(x_{0}\right)=-\delta$. If $\Sigma$ has a nondegenerate action spectrum, we can perturb explicitly the resulting Hamiltonian function near $\partial M$, so that all 1periodic orbits of $K_{\lambda}$ are nondegenerate (see [2]). Then we have

$$
I S_{k}^{[a, b[}(M)=\lim _{\lambda \rightarrow+\infty} S_{k}^{[a, b[}\left(K_{\lambda}\right),
$$

and each non-constant orbit of $K_{\lambda}$ has action near the period of a closed characteristic of $\partial M$. Moreover, the monotonicity morphism

$$
S_{k}^{[a, b[}\left(K_{\lambda_{1}}\right) \longrightarrow S_{k}^{[a, b[}\left(K_{\lambda_{2}}\right)
$$

is an isomorphism if $a \leqslant b<\lambda_{1} \leqslant \lambda_{2}$, because under the deformation $K_{\lambda_{1}} \rightsquigarrow K_{\lambda_{2}}$, no 1-periodic orbit appears below the level $\lambda_{1}$. This shows that the inductive limit morphism

$$
S_{k}^{[a, b[}\left(K_{\lambda_{0}}\right) \longrightarrow I S_{k}^{[a, b[}(M) \text { is an isomorphism if } b<\lambda_{0} .
$$

Observe that Floer trajectories can only connect orbits in the same homotopy class. We have therefore a splitting

$$
I S_{k}^{[a, b[}(M)=F H_{k}^{[a, b[}(M) \oplus N C_{k}^{[a, b[}(M),
$$

where $F H_{k}^{[a, b[}(H)$ is generated by the contractible 1-periodic orbits of the Hamiltonian function $H \in \mathcal{H}_{\mathrm{FH}}$. If $\varepsilon>0$ is small enough, we have

$$
I S_{k}^{[0, \varepsilon[}(M)=F H_{k}^{[0, \varepsilon[}(M)=H_{2 n-k}(M, \partial M)=H^{k}(M)
$$

and in particular for any $\lambda>\varepsilon$ we have

$$
S_{n}^{[0, \varepsilon[}\left(K_{\lambda}\right)=F H_{n}^{[0, \varepsilon[}\left(K_{\lambda}\right)=\mathbb{Z}_{2} \cdot x_{0}
$$

because the only orbits of $K_{\lambda}$ with small action are the constant orbits, which are contractible, and the Floer Homology of the Hamiltonian function $K_{\lambda}$ can be identified with the Morse homology of the function $-f$ on $M$.

BULletin DE LA SOCiÉtÉ MATHÉMATIQUE DE FRANCE 
3.2. The Floer-Hofer capacity. - A symplectic capacity issued from symplectic homology is defined in [5] as follows. Observe that if $B$ is the unit ball in $\mathbb{C}^{n}$, we have $\widehat{B}=\mathbb{C}^{n}$ and the almost complex structure $J_{0}=i$ fulfills the above assumptions. This means that $\mathcal{J}_{t}$ is the space of calibrated timedependent almost complex structures $J$ on $\mathbb{C}^{n}$ satisfying $J=i$ at infinity. The Liouville vector field associated to $\lambda_{0}$ is $\eta_{0}(z)=\frac{1}{2} z$, which leads to $\tau_{0}(z)=|z|^{2}$. The action spectrum of the unit sphere is $\{k \pi / k=1,2, \ldots\}$ : it is degenerate, but we remove the degeneracy if we replace the ball by a nearby irrational ellipsoid. For any bounded connected open set $U \subset \mathbb{C}^{n}$, set

$$
S_{k}^{[a, b[}(U)=S_{k}^{[a, b[}\left(\mathbb{C}^{n}, U\right)
$$

and observe that $\mathcal{H}_{\mathrm{FH}}(U)$ is a cofinal set in $\mathcal{H}_{\mathrm{QI}}(U)$. As above, we denote the open ball with radius $\sqrt{\rho}$ by $B^{\rho}$ and we have (see [5])

$$
\begin{array}{lll}
S_{n}^{[a, b]}\left(B^{\rho}\right)=\mathbb{Z}_{2} & \text { if } a \leqslant 0<b \leqslant \pi \rho, & S_{n}^{[a, b]}\left(B^{\rho}\right)=0 \text { else; } \\
S_{n+1}^{[a, b[}\left(B^{\rho}\right)=\mathbb{Z}_{2} & \text { if } 0<a \leqslant \pi \rho<b, & S_{n+1}^{[a, b[}\left(B^{\rho}\right)=0 \text { else; } \\
S_{k}^{[a, b]}\left(B^{\rho}\right)=0 & \text { if } k<n \text { or } n<k<3 n .
\end{array}
$$

Moreover, given any real numbers $r$ and $R$ such that $R>r>0$, the morphism $S_{k}^{[a, b[}\left(B^{R}\right) \rightarrow S_{k}^{[a, b[}\left(B^{r}\right)$ induced by the inclusion $B^{r} \subset B^{R}$ equals the identity as soon as both groups are isomorphic to $\mathbb{Z}_{2}$. Choose some open ball $B^{r}$ such that $B^{r} \subset U$ and let $\varepsilon$ be any real number satisfying $0<\varepsilon<\pi r$. For any real number $b>\pi r$, consider the inclusion morphism

$$
\sigma_{U}^{b}: S_{n+1}^{[\varepsilon, b[}(U) \longrightarrow S_{n+1}^{[\varepsilon, b[}\left(B^{r}\right)=\mathbb{Z}_{2},
$$

and define the capacity of $U$ by $c_{\mathrm{FHW}}(U)=\inf \left\{b / \sigma_{U}^{b}\right.$ is onto $\}$.

We will also consider another symplectic capacity issued from symplectic homology, which was already used in [7], and which is defined as follows. Observe that the ball satisfies the strong algebraic Weinstein conjecture in [16], which means that the natural map

$$
\mathbb{Z}_{2}=S_{n}^{[0, \varepsilon[}(B) \longrightarrow S_{n}^{[0, b[}(B) \text { vanishes for large enough } b .
$$

If $R$ is large enough, we have $B^{r} \subset U \subset B^{R}$, which defines inclusion morphisms

$$
\mathbb{Z}_{2}=S_{n}^{[0, \varepsilon[}\left(B^{R}\right) \stackrel{i_{R}}{\longrightarrow} S_{n}^{[0, \varepsilon[}(U) \stackrel{i_{r}}{\longrightarrow} S_{n}^{[0, \varepsilon[}\left(B^{r}\right)=\mathbb{Z}_{2} .
$$

Since $i_{r} \circ i_{R}$ is an isomorphism, we have $\alpha_{U}=i_{R}(1) \neq 0$. Consider the natural map

$$
i_{U}^{b}: S_{n}^{[0, \varepsilon[}(U) \longrightarrow S_{n}^{[0, b[}(U),
$$

and set $c_{\mathrm{FH}}(U)=\inf \left\{b / i_{U}^{b}\left(\alpha_{U}\right)=0\right\}$. In some sense, the capacity $c_{\mathrm{FHW}}$ measures a set from inside, whereas $c_{\mathrm{FH}}$ measures it from outside.

TOME $132-2004-\mathrm{N}^{\mathrm{O}} 4$ 
Proposition 3.2. - The maps $c_{\mathrm{FHW}}$ and $c_{\mathrm{FH}}$ are symplectic capacities and we have

$$
c_{\mathrm{FHW}} \leqslant c_{\mathrm{FH}} .
$$

Moreover, if $U$ is a RCT open set, then we have $c_{\mathrm{FHW}}(U)=c_{\mathrm{FH}}(U)$.

Proof. - It is proved in [5] that $c_{\mathrm{FHW}}$ is a capacity, and the same proof works in the case of $c_{\mathrm{FH}}$ : the independence of the choices involved and the invariance by $\mathcal{D}$ are due to the Isotopy Invariance Theorem, the monotonicity comes from the functoriality of the inclusion morphisms, the homogeneity results from (3.5), and the normalization comes from the computation of the symplectic homology groups of ellipsoids. Consider now the following commutative diagram

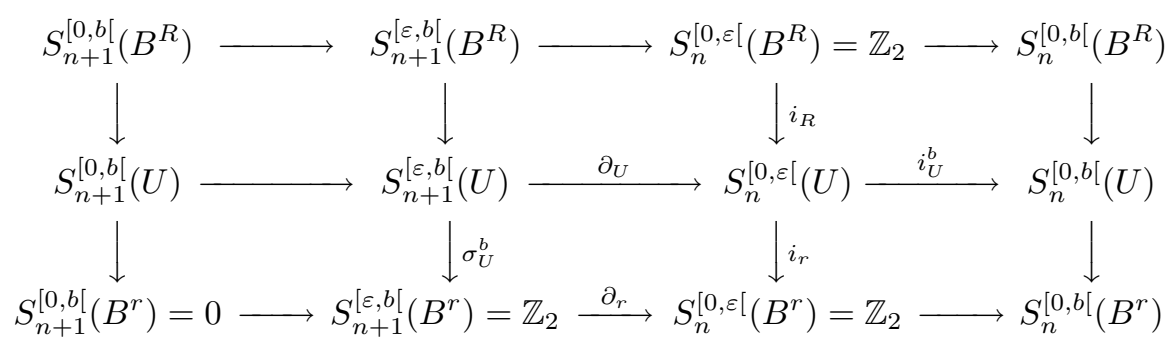

where the horizontal arrows are the exact triangles $\Delta^{0, \varepsilon, b}$, and the vertical arrows are the inclusion morphisms. Notice that $i_{r}\left(\alpha_{U}\right)=1$ and that $\partial_{r}$ is an isomorphism. If we assume that $i_{U}^{b}\left(\alpha_{U}\right)=0$, the exactness of the lines shows that there exists some $\beta \in S_{n+1}^{[\varepsilon, b[}(U)$ satisfying $\alpha_{U}=\partial_{U}(\beta)$, which leads to $\partial_{r}\left(\sigma_{U}^{b}(\beta)\right)=1$, and $\sigma_{U}^{b}$ is onto. This proves that $c_{\mathrm{FHW}}(U) \leqslant c_{\mathrm{FH}}(U)$. Moreover, if $U$ is a RCT open set, the computations in [7] show that $S_{n}^{[0, \varepsilon[}(U)=\mathbb{Z}_{2} \cdot \alpha_{U}$ for small enough $\varepsilon$ (see Section 5.2), and $i_{r}$ is an isomorphism. This shows that the map $\sigma_{U}^{b}$ is onto if and only if $i_{U}^{b}\left(\alpha_{U}\right)=0$, which implies the equality.

The above capacities can also be defined in the general framework of Hamiltonian capacities from Section 2: we will deal with the capacity $c_{\mathrm{FH}}$, the case of $c_{\mathrm{FHW}}$ being very similar. The main point is to make sure that the orbit we are looking for exists. Consider the class $\mathcal{H}_{\mathrm{FH}}^{\mathrm{c}}$ of those Hamiltonian functions $H \in \mathcal{H}_{\mathrm{FH}}$ satisfying $H>-\pi r$ on $S^{1} \times \mathbb{C}^{n}$ and $H<0$ near some open ball $B^{r}$ with radius $\sqrt{r}$ and moreover $H=\mu \tau_{0}+c$ at infinity, where $\mu>\pi$. Observe that $\mathcal{H}_{\mathrm{FH}}^{\mathrm{c}}(U)=\mathcal{H}_{\mathrm{FH}}(U) \cap \mathcal{H}_{\mathrm{FH}}^{\mathrm{c}}$ is a cofinal set in $\mathcal{H}_{\mathrm{QI}}(U)$. Given $H \in \mathcal{H}_{\mathrm{FH}}$, choose any real number $\delta$ satisfying $-\min H<\delta<\pi r$ and consider the function $f=-\delta+\nu \tau_{0}$, where $\nu>0$. If $\nu$ is small enough, we have $f \in \mathcal{H}_{\mathrm{FH}}\left(B^{r}\right)$ and $H(t, \cdot) \geqslant f$ on $\mathbb{C}^{n}$ (see Figure 2). Choose any real number $\varepsilon$ such that $\delta<\varepsilon<\pi r$ and consider the monotonicity morphism

$$
\sigma_{f}: \mathbb{Z}_{2} \cdot x_{0}=S_{n}^{[0, \varepsilon[}(f) \longrightarrow S_{n}^{[0, \varepsilon[}(H)
$$

BULletin DE LA SOCiÉtÉ MATHÉMATIQUE DE FRANCE 


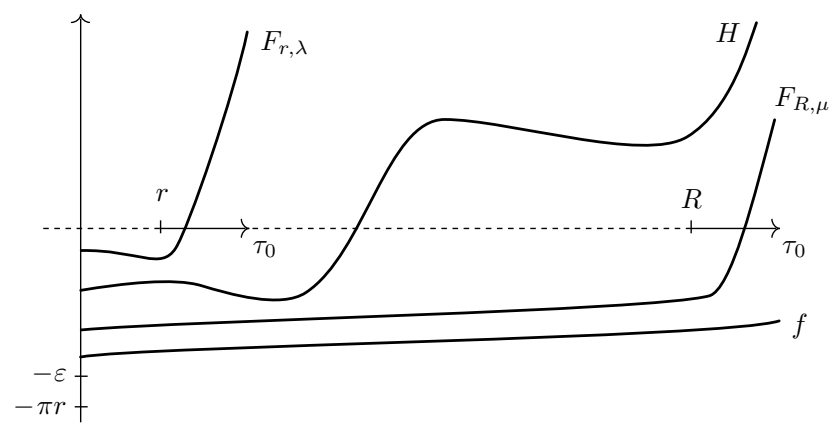

FiguRE 2. The Floer-Hofer selector: $f \prec F_{R, \mu} \prec H \prec F_{r, \lambda}$

where $x_{0}$ is the center of the ball $B^{r}$, and set $\alpha_{H}=\sigma_{f}\left(x_{0}\right)$. Consider the natural map

$$
i_{H}^{b}: S_{n}^{[0, \varepsilon[}(H) \longrightarrow S_{n}^{[0, b[}(H),
$$

and define the energy of $H$ by $c_{\mathrm{FH}}(H)=\inf \left\{b / i_{H}^{b}\left(\alpha_{H}\right)=0\right\}$.

Proposition 3.3. - The energy $c_{\mathrm{FH}}$ satisfies:

(i) for each $H \in \mathcal{H}_{\mathrm{FH}}^{\mathrm{c}}$, the number $c_{\mathrm{FH}}(H)$ is a critical value of $A_{H}$;

(ii) $c(\alpha \star H \circ \phi)=\alpha c(H)$ for any $\phi \in \mathcal{D}$ and any $\alpha>0$;

(iii) if $H_{1} \leqslant H_{2}$ on $S^{1} \times \mathbb{C}^{n}$, then $c_{\mathrm{FH}}\left(H_{1}\right) \geqslant c_{\mathrm{FH}}\left(H_{2}\right)$;

(iv) $c_{\mathrm{FH}}(U)=\inf \left\{c_{\mathrm{FH}}(H) / H \in \mathcal{H}_{\mathrm{FH}}^{\mathrm{c}}(U)\right\}$.

Proof. - As above, the definition of the energy $c_{\mathrm{FH}}(H)$ does not depend of the choice of $f$. The main point is to prove that $\alpha_{H}$ is nonzero and that $i_{H}^{b}\left(\alpha_{H}\right)=0$ for large enough $b$. As in (3.6), consider the autonomous function $F_{r, \lambda}$, where

$$
F_{r, \lambda}= \begin{cases}-\delta_{1}+\nu_{1}\left(\tau_{0}-r\right) & \text { in } B^{r} \\ -\delta_{1}+\lambda\left(\tau_{0}-r\right) & \text { off } B^{r}\end{cases}
$$

for small enough positive numbers $\delta_{1}, \nu_{1}$ and large enough $\lambda$. After perturbation, we get a Hamiltonian function $F_{r, \lambda} \in \mathcal{H}_{\mathrm{FH}}\left(B^{r}\right)$, whose Floer homology coincides with the symplectic homology of $B^{r}$ below the level $\lambda r$, up to a shift of $\delta_{1}$. Similarly, choose a real number $R>r$ such that $H=\mu \tau_{0}+c$ off $B^{R}$, and perturb the autonomous function given by

$$
F_{R, \mu}= \begin{cases}-\delta_{2}+\nu_{2}\left(\tau_{0}-R\right) & \text { in } B^{R} \\ -\delta_{2}+\mu\left(\tau_{0}-R\right) & \text { off } B^{R}\end{cases}
$$

TOME $132-2004-\mathrm{N}^{\mathrm{O}} 4$ 
into $F_{R, \mu} \in \mathcal{H}_{\mathrm{FH}}\left(B^{R}\right)$. We can choose the parameters so that $f \leqslant F_{R, \mu} \leqslant H \leqslant$ $F_{r, \lambda}$ on $S^{1} \times \mathbb{C}^{n}$ (see Figure 2 ). Then we have a commutative diagram

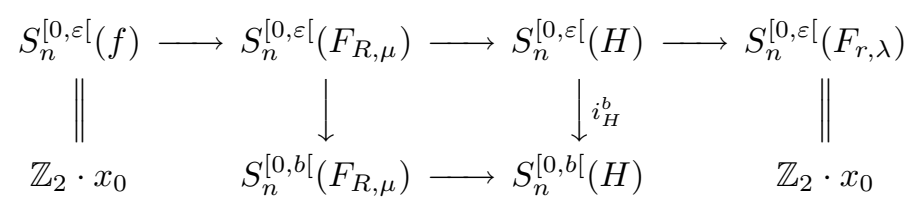

and the monotonicity morphism $S_{n}^{[0, \varepsilon[}(f) \rightarrow S_{n}^{[0, \varepsilon[}\left(F_{r, \lambda}\right)$ factorizes by $\sigma_{f}$. Since this map is an isomorphism, this shows that $\alpha_{H}=\sigma_{f}\left(x_{0}\right)$ is nonzero. Moreover, since $\mu>\pi$, we have

$$
S_{n}^{[0, b[}\left(F_{R, \mu}\right)=0 \text { for large enough } b,
$$

which proves that $i_{H}^{b}\left(\alpha_{H}\right)=0$. The rest of the proof of Proposition 3.3 is straightforward.

\section{Generating functions}

Here we recall the definition of the Viterbo capacity (see [14]) and the uniqueness of symplectic homology proved in [15], which is the main tool in the proof of our results. We warn the reader that our signs conventions are not the one in [14], because we are looking for critical values of $A_{H}$ instead of $-A_{H}$.

4.1. The Viterbo capacity. - Let $\mathcal{H}_{\mathrm{V}} \subset \mathcal{H}_{\mathrm{t}}$ be the class of those compactly supported Hamiltonian functions. Given any $H \in \mathcal{H}_{\mathrm{V}}$, consider the Lagrangian graph of $\phi=\phi_{1}^{H}$ given by

$$
\Gamma_{\phi}=\left\{(z, \phi(z)) / z \in \mathbb{C}^{n}\right\} \subset\left(\mathbb{C}^{n},-\omega\right) \times\left(\mathbb{C}^{n}, \omega\right) \simeq T^{*} \Delta,
$$

where $\Delta$ is the diagonal and the last identification is given by the symplectomorphism

$$
(z, Z) \longmapsto\left(\frac{1}{2}(z+Z), i(Z-z)\right) .
$$

Since $\Gamma_{\phi}$ coincides with $\Delta$ off a compact set, we can add a point at infinity and get a compact Lagrangian submanifold $\Gamma$ in $T^{*} S^{2 n}$ which is Hamiltonian isotopic to the zero section. By a theorem of Sikorav, $\Gamma$ has a generating function $S: S^{2 n} \times \mathbb{R}^{N} \rightarrow \mathbb{R}$ which is quadratic at infinity. This means that 0 is a regular value of the fiber derivative $\partial_{\xi} S$ of $S$, that we have

$$
\Gamma=\left\{\left(q, \partial_{q} S(q, \xi)\right) /(q, \xi) \in S^{2 n} \times \mathbb{R}^{N} \text { satisfies } \partial_{\xi} S(q, \xi)=0\right\},
$$

and that we have $S(q, \xi)=Q(\xi)$ off a compact set, where $Q$ is some nondegenerate quadratic form on $\mathbb{R}^{N}$. If we fix the critical value of $S$ at infinity by setting $S\left(\infty, \xi_{\infty}\right)=0$, the function $S$ can be seen as a finite-dimensional reduction of the action functional $A_{H}$. More precisely, the critical points $(q, \xi)$ for $S$ are in 1-1 correspondence with the 1-periodic orbits $\gamma$ for $H$ and we have 
$A_{H}(\gamma)=S(q, \xi)$. If $(q, \xi)$ is a Morse critical point of $S$, then $\gamma$ is a nondegenerate orbit of $H$, and its Conley-Zehnder index satisfies

$$
i_{\mathrm{CZ}}(H ; \gamma)=i_{M}(S ; q, \xi)-n-i,
$$

where $i$ denotes the index of $Q$ (see [14], [10] and compare (3.2)). Set

$$
S^{\lambda}=\left\{(q, \xi) \in S^{2 n} \times \mathbb{R}^{N} / S(q, \xi)<\lambda\right\} .
$$

It is proved in [14] and in [11] that the relative homology groups $H_{*+i}\left(S^{\beta}, S^{\alpha}\right)$ depend only on $\Gamma$. These groups have been used in [12] as a substitute of symplectic homology. By the Thom isomorphism, we have $H_{*}\left(S^{\alpha}, S^{-\alpha}\right)=$ $H_{*}\left(S^{2 n}\right) \otimes H_{*}\left(D^{i}, S^{i-1}\right)$ for large enough $\alpha$. Consider the map

$$
j_{S}^{\lambda}: H_{*+i}\left(S^{\alpha}, S^{-\alpha}\right) \longrightarrow H_{*+i}\left(S^{\alpha}, S^{\lambda}\right)
$$

induced by inclusion, where $*=0,2 n$. Using Lusternik-Schnirelman theory, we obtain critical values of $S$ by setting

$$
c_{+}(\phi)=\inf \left\{\lambda / j_{S}^{\lambda}(\mu)=0\right\} \quad \text { and } \quad c_{-}(\phi)=\inf \left\{\lambda / j_{S}^{\lambda}(1)=0\right\},
$$

where we identify $\alpha$ with $\alpha \otimes 1$ and where $\mu$ is a generator of $H_{2 n}\left(S^{2 n}\right)$. Then we have

$$
c_{-}(\phi) \leqslant 0 \leqslant c_{+}(\phi) \text { and } c_{-}(\phi)=c_{+}(\phi)=0 \Longleftrightarrow \phi=\mathrm{id} .
$$

In particular, if $H$ is $C^{2}$-small and autonomous, then $\Gamma$ has a generating function on $S^{2 n}$ having the same critical points as the function $-H$ on $\mathbb{C}^{n}$. We get in this case

$$
c_{+}(\phi)=-\min _{z \in \mathbb{C}} H(z) \quad \text { and } \quad c_{-}(\phi)=-\max _{z \in \mathbb{C}} H(z) .
$$

Notice that we can recover the signs in [14] as follows. Consider the function $\bar{S}=-S$ and the natural maps

$$
i \frac{\lambda}{S}: H^{*+\bar{\imath}}\left(\bar{S}^{\alpha}, \bar{S}^{-\alpha}\right) \rightarrow H^{*+\bar{\imath}}\left(\bar{S}^{\lambda}, \bar{S}^{-\alpha}\right),
$$

where $\bar{\imath}$ is the index of $\bar{S}$. The original invariants $c_{ \pm}$in [14] are defined by

$$
\bar{c}_{+}(\phi)=\sup \left\{\lambda / i \frac{\lambda}{\bar{S}}(\bar{\mu})=0\right\} \quad \text { and } \quad \bar{c}_{-}(\phi)=\sup \left\{\lambda / i_{\bar{S}}^{\lambda}(\overline{1})=0\right\}
$$

where $\overline{1}, \bar{\mu}$ generate $H^{0}\left(S^{2 n}\right)$ and $H^{2 n}\left(S^{2 n}\right)$. Since $\bar{S}^{\lambda}$ is the complement of $S^{\lambda}$ in $S^{2 n} \times \mathbb{R}^{i+\bar{\imath}}$, we get by Alexander duality

$$
c_{+}(\phi)=-\bar{c}_{-}(\phi)=\bar{c}_{+}\left(\phi^{-1}\right) \text { and } c_{-}(\phi)=-\bar{c}_{+}(\phi)=\bar{c}_{-}\left(\phi^{-1}\right) .
$$

Moreover, if $H_{1} \leqslant H_{2}$ on $S^{1} \times \mathbb{C}^{n}$, then $c_{ \pm}\left(\phi_{1}\right) \geqslant c_{ \pm}\left(\phi_{2}\right)$. In particular, if $H$ is nonpositive, then $c_{-}(\phi)=0$, and if $H$ is nonnegative, then $c_{+}(\phi)=0$. However, we will be particularly interested in the following slightly different situation. Define a partial ordering $\sqsubset$ on $\mathcal{H}_{\mathrm{V}}$ by (see [12])

$$
H \sqsubset K \Longleftrightarrow \phi_{1}^{K} \circ\left(\phi_{1}^{H}\right)^{-1} \text { is the time } 1 \text { flow of } L \geqslant 0 \text { on } S^{1} \times \mathbb{C}^{n} .
$$

TOME $132-2004-\mathrm{N}^{\mathrm{O}} 4$ 
If $H_{1} \sqsubset H_{2}$, there exists two gfqi $S_{1}$ of $\Gamma_{1}$ and $S_{2}$ of $\Gamma_{2}$ having the same quadratic form at infinity satisfying $S_{2} \leqslant S_{1}$ on $S^{2 n} \times \mathbb{R}^{N}$. The inclusion $S_{1}^{\lambda} \subset S_{2}^{\lambda}$ defines a map

$$
i\left(S_{1}, S_{2}\right): H_{k}\left(S_{1}^{\beta}, S_{1}^{\alpha}\right) \longrightarrow H_{k}\left(S_{2}^{\beta}, S_{2}^{\alpha}\right) .
$$

We obtain this way a commutative diagram, which implies that we have $c_{+}\left(\phi_{2}\right) \leqslant c_{+}\left(\phi_{1}\right)$ and $c_{-}\left(\phi_{2}\right) \leqslant c_{-}\left(\phi_{1}\right)$. It is important to notice that, even if $\sqsubset$ is not the natural partial ordering $\prec$ in Section 2 , the cofinal sequences are the same for both partial orderings (see [12], Remark 5.7). In other terms, in the direct limit process, we can forget the difference between $\sqsubset$ and $\prec$. The Viterbo capacity of $U$ is defined by

$$
c_{\mathrm{V}}(U)=\sup \left\{c_{+}\left(\phi_{1}^{H}\right) / \operatorname{Supp}(H) \subset S^{1} \times U\right\} .
$$

It fits into case (CS) of the settings in Section 2: the admissible class $\mathcal{H}_{\mathrm{V}}(U)$ is the set of Hamiltonian functions with support in $S^{1} \times U$, and the critical value is $c_{\mathrm{V}}(H)=c_{+}\left(\phi_{1}^{H}\right)$. For any cofinal family $H_{\lambda}$ in $\mathcal{H}_{\mathrm{V}}(U)$ satisfying

$$
\forall H \in \mathcal{H}_{\mathrm{V}}(U), \exists A \in \mathbb{R} \text { such that } \lambda>A \Rightarrow H_{\lambda} \leqslant H \text { on } S^{1} \times \mathbb{C}^{n}
$$

(which is cofinal for $\sqsupset$ by the above remark), we have $c_{\mathrm{V}}(U)=\lim _{\lambda \rightarrow+\infty} c_{\mathrm{V}}\left(H_{\lambda}\right)$. The homogeneity of $c_{\mathrm{V}}$ is due to the fact that $r^{2} \star S$ is a generating function for the graph of the time 1 flow of $r^{2} \star H$, and the symplectic invariance results from the identity $c_{ \pm}\left(\phi^{-1} \psi \phi\right)=c_{ \pm}(\psi)$ (see [14]). It is important to notice that the proof of this identity shows that the map $H \mapsto c_{\mathrm{V}}(H)$ is continuous with respect to the $C^{0}$-topology on $\mathcal{H}_{\mathrm{V}}$.

For later purpose, it will be useful to characterize $c_{\mathrm{V}}(H)$ in terms of relative homology of finite positive sublevels of $S$. In this aim, consider the class $\mathcal{H}_{\mathrm{V}}^{\mathrm{c}} \subset \mathcal{H}_{\mathrm{V}}$ of those compactly supported Hamiltonian functions satisfying

$$
H \leqslant 0 \text { on } S^{1} \times \mathbb{C}^{n} \text { and } H<0 \text { in } S^{1} \times B^{r}
$$

where $B$ is some nonempty open ball with radius $\sqrt{r}$, and observe that $\mathcal{H}_{\mathrm{V}}^{\mathrm{c}}(U)$ is a cofinal set in $\mathcal{H}_{\mathrm{V}}(U)$. Given $H \in \mathcal{H}_{\mathrm{V}}^{\mathrm{c}}$, let $f_{0}$ be some $C^{2}$-small autonomous Hamiltonian function having a unique minimum satisfying

$$
H \sqsubset f_{0}, \quad H(t, .) \leqslant f_{0} \leqslant 0 \text { on } \mathbb{C}^{n} \text { and } f_{0}<0 \text { in } B^{r}
$$

(see Figure 3). Let $S$ and $S_{0}$ be generating functions associated to $H$ and $f_{0}$ having the same quadratic form at infinity and satisfying $S_{0} \leqslant S$ on $S^{2 n} \times \mathbb{R}^{N}$, and choose $0<\eta<-\min \left(f_{0}\right)$. We get the following characterization of $c_{\mathrm{V}}(H)$.

Proposition 4.1. - If $H$ is an element of $\mathcal{H}_{\mathrm{V}}^{\mathrm{c}}$ and if $\eta \leqslant a<c_{\mathrm{V}}(H)<b \leqslant c$, then $H_{2 n+i}\left(S^{b}, S^{\eta}\right)$ contains an element $\gamma_{H}$ with the following properties:

(i) $\sigma_{S}^{b}\left(\gamma_{H}\right) \neq 0$, where $\sigma_{S}^{b}: H_{2 n+i}\left(S^{b}, S^{\eta}\right) \rightarrow H_{2 n+i}\left(S_{0}^{b}, S_{0}^{\eta}\right)$ is the natural map; 


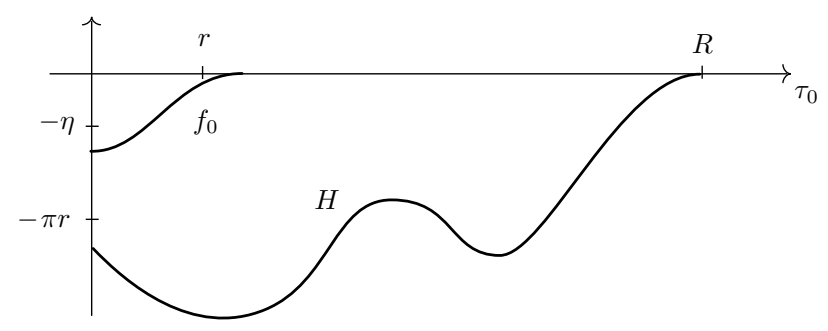

Figure 3. The Viterbo selector: $H \sqsubset f_{0} \sqsubset 0$

(ii) if $j_{b}^{a}: H_{2 n+i}\left(S^{b}, S^{\eta}\right) \rightarrow H_{2 n+i}\left(S^{b}, S^{a}\right)$ is the natural map, then $\gamma_{a}=$ $j_{b}^{a}\left(\gamma_{H}\right)$ satisfies $i_{S}^{c}\left(\gamma_{a}\right) \neq 0$, where $i_{S}^{c}$ is the natural map

$$
H_{2 n+i}\left(S^{b}, S^{a}\right) \longrightarrow H_{2 n+i}\left(S^{c}, S^{a}\right) .
$$

Proof. - Remark first that $c_{\mathrm{V}}(H) \geqslant c_{\mathrm{V}}\left(f_{0}\right)=-\min \left(f_{0}\right)>\eta$, and consider the diagram

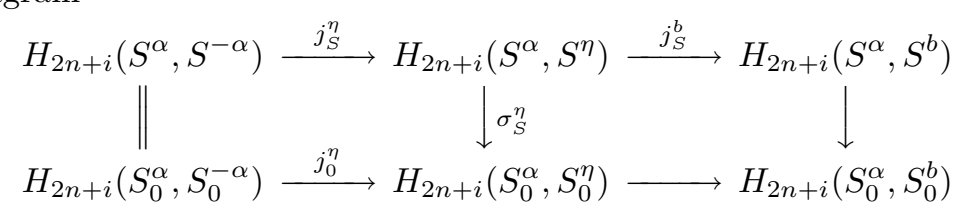

where all the arrows are natural and where $\alpha$ is large enough. We see that $\gamma=j_{S}^{\eta}(\mu) \neq 0$ and $\sigma_{S}^{\eta}(\gamma)=j_{0}^{\eta}(\mu) \neq 0$. Moreover, we know that $j_{S}^{b}(\gamma)=0$ for $b>c_{\mathrm{V}}(H)$. Consider now the commutative diagram

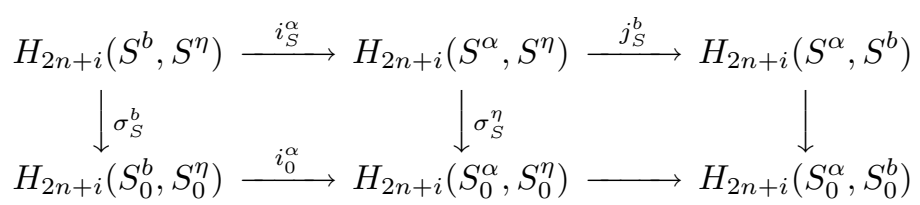

where the lines are exact sequences. There exists $\gamma_{H} \in H_{2 n+i}\left(S^{b}, S^{\eta}\right)$ satisfying $i_{S}^{\alpha}\left(\gamma_{H}\right)=\gamma$. We infer $i_{0}^{\alpha}\left(\sigma_{S}^{b}\left(\gamma_{H}\right)\right)=\sigma_{S}^{\eta}(\gamma) \neq 0$, which proves (i).

In the commutative diagram

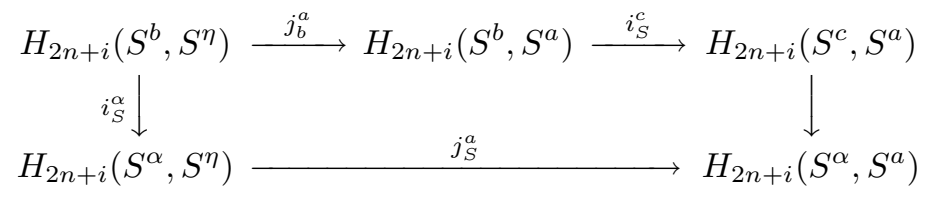

we know that $j_{S}^{a}\left(i_{S}^{\alpha}\left(\gamma_{H}\right)\right)=j_{S}^{a}(\gamma) \neq 0$, because we assume $a<c_{\mathrm{V}}(H)$. This proves that $i_{S}^{c}\left(\gamma_{a}\right)=i_{S}^{c}\left(j_{b}^{a}\left(\gamma_{H}\right)\right)$ is nonzero, which proves (ii).

TOME $132-2004-\mathrm{N}^{\mathrm{O}} 4$ 
REMARK 4.2. - The above functorial properties allow to define several other symplectic capacities. First, with the notations of Proposition 4.1, define

$$
c_{\mathrm{I}}(H)=\inf \left\{b / \sigma_{S}^{b} \text { is onto }\right\} \text { and } c_{\mathrm{I}}(U)=\sup \left\{c_{\mathrm{I}}(H) / \operatorname{Supp}(H) \subset S^{1} \times U\right\} .
$$

On the other hand, consider the inclusion morphism

$$
\tau_{U}^{b}: \mathbb{Z}_{2}=S_{n+1}^{[b,+\infty[}\left(B^{R}\right) \rightarrow S_{n+1}^{[b,+\infty[}(U)
$$

and set $c_{\mathrm{O}}(U)=\inf \left\{b / \tau_{U}^{b}(1)=0\right\}$. Our proof shows that we have

$$
\stackrel{\vee}{c}_{\mathrm{FHW}} \leqslant{\stackrel{\vee}{c_{\mathrm{FH}}}} \leqslant \stackrel{\vee}{c}_{\mathrm{O}} \leqslant c_{\mathrm{I}} \leqslant c_{\mathrm{V}}
$$

and that all these capacities are equal for RCT open sets.

4.2. Uniqueness of symplectic homology. - Given $H \in \mathcal{H}_{\mathrm{V}}^{\mathrm{c}}$, consider a generating function $S$ of $\Gamma$ as above: we have on one hand the relative homology groups $H_{*}\left(S^{\beta}, S^{\alpha}\right)$. On the other hand, if we assume that all 1-periodic orbits in the support of $H$ are nondegenerate and that $0 \notin[a, b[$, we have well-defined Floer homology groups $S_{*}^{[a, b[}(H)$. The uniqueness of symplectic homology proved in in [15] can be expressed as the following theorem.

TheOrem 4.3. - We have $S_{k}^{[a, b[}(H)=H_{k+n+i}\left(S^{b}, S^{a}\right)$ if $0 \notin[a, b[$.

The difference in indices is explained by (4.1). Moreover, the proof in [15] is based on the Floer homology of the Hamiltonian function $S$, which is some kind of interpolation between its Morse homology and the Floer homology of $H$. This shows that all the above maps in symplectic homology identify with natural maps in the homology of generating functions. In particular, given two Hamiltonians $H_{1}$ and $H_{2}$ with $H_{1} \sqsubset H_{2}$ and $H_{1} \prec H_{2}$, we have on one hand the natural map (4.3), and on the other hand the monotonicity morphism given by (3.4), and the proof of Theorem 4.3 shows that these maps are the same.

THEOREM 4.4. - If $0 \notin[a, b[$, there is a commutative diagram

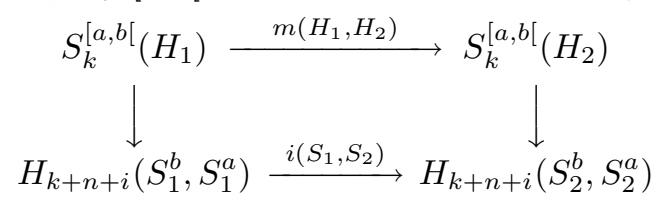

where the vertical arrows are the isomorphisms in Theorem 4.3.

We can now rephrase Proposition 4.1 as follows.

Proposition 4.5. - Given $H \in \mathcal{H}_{\mathrm{V}}^{\mathrm{c}}$ and $f_{0}$ as in (4.4), if $0<\eta<-\min f_{0}$ and $\eta \leqslant a<c_{\mathrm{V}}(H)<b$, then $S_{n}^{[\eta, b[}(H)$ contains an element $\gamma_{H}$ satisfying

(i) $\sigma_{H}^{b}\left(\gamma_{H}\right) \neq 0$, where $\sigma_{H}^{b}: S_{n}^{[\eta, b[}(H) \rightarrow S_{n}^{[\eta, b[}\left(f_{0}\right)$ is the monotonicity morphism.

BULletin DE LA SOCIÉtÉ MATHÉMATIQUE DE FRANCE 


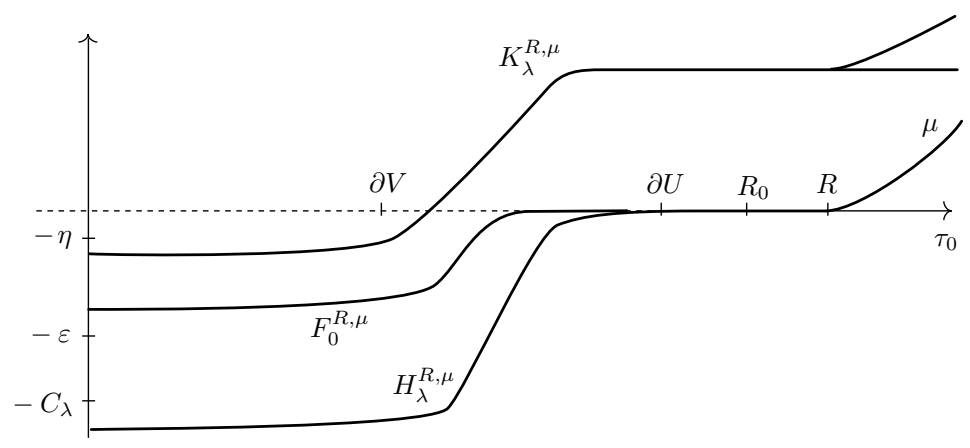

Figure 4. $H_{\lambda}^{R, 0}=H_{\lambda} \prec f_{0}=F_{0}^{R, 0} \prec F_{0}^{R, \mu} \prec K_{\lambda}^{R, \mu}=H_{\lambda}^{R, \mu}+C_{\lambda}$

(ii) Set $\gamma_{a}=j^{a}\left(\gamma_{H}\right)$, where $j^{a}: S_{n}^{[\eta, b[}(H) \rightarrow S_{n}^{[a, b[}(H)$ is the natural map. For any $c>b$, we have $i^{c}\left(\gamma_{a}\right) \neq 0$, where $i^{c}$ is the natural map

$$
S_{n}^{[a, b[}(H) \longrightarrow S_{n}^{[a, c[}(H) .
$$

If we had considered the same type of Hamiltonian functions, Theorems 4.3 and 4.4 would mean that, after excision of a neighborhood of the point at infinity, the generating functions homology in [12] coincides with symplectic homology, and the capacities $c_{\mathrm{V}}$ and $\check{c}_{\mathrm{FH}}$ would be equal. However, we have first to turn a compactly supported Hamiltonian function $H$ into a function which is quadratic at infinity. For this, choose large enough real numbers $R$ and $\mu$ satisfying $H \in \mathcal{H}_{\mathrm{V}}\left(B^{R}\right), \mu>0$ and $\mu \notin \pi \mathbb{Z}$, and deform $H^{R, 0}=H$ into $H^{R, \mu}$ satisfying

$$
H^{R, \mu}= \begin{cases}H & \text { on } S^{1} \times B^{R}, \\ \mu\left(\tau_{0}-R\right) & \text { off } B^{R+\nu},\end{cases}
$$

where $\nu$ is small enough and all 1-periodic orbits of $H^{R, \mu}$ with nonzero action are nondegenerate (see Figure 4 ). This creates periodic orbits with action at least $\pi R$. As a consequence, we get the following lemma.

LEMMA 4.6. - If we have $0<a \leqslant b<\pi R$, then the monotonicity morphism

$$
m\left(H, H^{R, \mu}\right): S_{k}^{[a, b[}(H) \longrightarrow S_{k}^{[a, b[}\left(H^{R, \mu}\right) \text { is an isomorphism. }
$$

This allows to see the difference between symplectic homology and generating functions homology in the following way. Start with a cofinal family $H_{\lambda}$ in $\mathcal{H}_{\mathrm{V}}(U)$ such that $H_{\lambda} \approx-C_{\lambda}$ inside $S^{1} \times U$, where $C_{\lambda}$ is some large constant, and deform each $H_{\lambda}$ into $H_{\lambda}^{R, \mu}$ as in (4.5), where $R$ and $\mu$ go to infinity with $\lambda$. By the above results, the generating functions homology of $U$ identifies with

$$
\begin{aligned}
G F_{n+k}^{[a, b[}(U) & =\longleftarrow \lim _{k+n+i}\left(S_{\lambda}^{b}, S_{\lambda}^{a}\right) \\
& =\lim _{\lambda \rightarrow+\infty} S_{k}^{[a, b[}\left(H_{\lambda}^{R, \mu}\right) \text { if } 0<a \leqslant b<+\infty,
\end{aligned}
$$

TOME $132-2004-\mathrm{N}^{\mathrm{O}} 4$ 
and it can be seen as a symplectic homology with compact support. On the other hand, if $\bar{V} \subset U$, we can choose the parameters so that $K_{\lambda}=H_{\lambda}^{R, \mu}+C_{\lambda}$ is a cofinal family in $\mathcal{H}_{\mathrm{FH}}(V)$, and we get

$$
S_{k}^{[a, b[}(V)=\lim _{\lambda \rightarrow+\infty} S_{k}^{[a, b[}\left(H_{\lambda}^{R, \mu}+C_{\lambda}\right)=\lim _{\lambda \rightarrow+\infty} S_{k}^{\left[a+C_{\lambda}, b+C_{\lambda}[\right.}\left(H_{\lambda}^{R, \mu}\right) .
$$

This difference between compactly supported and quadratic at infinity Hamiltonian functions is the main object of this paper, and will be studied in the next section.

\section{Proofs of the main results}

5.1. Comparison of the capacities. - Let $U$ and $V$ be two bounded connected open sets in $\mathbb{C}^{n}$ such that $\bar{V} \subset U$. Assume that $U$ and $V$ are isotopic to each other: we will prove that $c_{\mathrm{FH}}(V) \leqslant c_{\mathrm{V}}(U)$, which implies Theorem 1.4. Fix some real numbers $R_{0}$ and $r>0$ such that $B^{r} \subset V$ and $\bar{U} \subset B^{R_{0}}$, and consider a cofinal family of Hamiltonian functions $H_{\lambda} \in \mathcal{H}_{\mathrm{V}}^{\mathrm{c}}(U)$ satisfying

$$
-C_{\lambda}-2 \delta<H_{\lambda}<-C_{\lambda}-\delta \text { in } S^{1} \times \bar{V} \text { and } \operatorname{Supp}\left(H_{\lambda}\right) \subset S^{1} \times U,
$$

where the constant $\delta=\delta_{\lambda}$ goes to zero and $C_{\lambda}$ goes to infinity with $\lambda$. We know that

$$
c_{\mathrm{V}}(U)=\lim _{\lambda \rightarrow+\infty} c_{\mathrm{V}}\left(H_{\lambda}\right) .
$$

Moreover, we can assume that all 1-periodic orbits of $H_{\lambda}$ with nonzero action are nondegenerate. Consider now a $C^{2}$-small compactly supported function $f_{0}$ on $\mathbb{C}^{n}$ such that

$$
-3 \delta<f_{0}<-2 \delta \text { in } \bar{V} \text { and } \operatorname{Supp}\left(f_{0}\right) \subset U,
$$

which coincides inside $\operatorname{Supp}\left(f_{0}\right)$ with a Morse function having a unique local minimum $x_{0}$ (this is the reason for assuming that $U$ and $V$ are isotopic to each other). Choose now real numbers $R>R_{0}$ and $\mu \notin \pi \mathbb{Z}$ going to infinity with $\lambda$, and deform $H_{\lambda}=H_{\lambda}^{R, 0}$ into $H_{\lambda}^{R, \mu}$ and $f_{0}=F_{0}^{R, 0}$ into $F_{0}^{R, \mu}$ as in (4.5). We have

$$
H_{\lambda}^{R, \mu} \leqslant F_{0}^{R, \mu} \leqslant K_{\lambda}^{R, \mu}=H_{\lambda}^{R, \mu}+C_{\lambda} \quad \text { on } \quad S^{1} \times \mathbb{C}^{n}
$$

(see Figure 4). If we choose $R$ and $\mu$ such that $\mu R \leqslant \frac{1}{2} C_{\lambda}$, then $K_{\lambda}^{R, \mu}$ is a cofinal family in $\mathcal{H}_{F H}(V)$. Indeed, we have $K_{\lambda}^{R, \mu} \geqslant C_{\lambda}$ off $U$ and $C_{\lambda} / R \geqslant 2 \mu$ goes to infinity with $\lambda$, and moreover the only degenerate orbits of $K_{\lambda}^{R, \mu}$ have action $-C_{\lambda}$, which goes to $-\infty$, and we only consider orbits whose action is bounded from below, that is, we always require $a>-\infty$. Choose now any level $b$ such that $c_{\mathrm{V}}(U)<b<\pi R_{0}$, and consider the monotonicity morphisms

$$
S_{n}^{[\eta, b[}\left(H_{\lambda}^{R, \mu}\right) \stackrel{I_{\lambda}^{b}}{\longrightarrow} S_{n}^{[\eta, b[}\left(F_{0}^{R, \mu}\right)=\mathbb{Z}_{2} \cdot x_{0} \stackrel{J_{\lambda}^{b}}{\longrightarrow} S_{n}^{[\eta, b[}\left(K_{\lambda}^{R, \mu}\right)
$$

BULLETIN DE LA SOCIÉtÉ MATHÉMATIQUE DE FRANCE 
where $0<\eta<\delta$. We know by Proposition 4.5 and Lemma 4.6 that $I_{\lambda}^{b}$ is onto. On the other hand, we have $K_{\lambda}^{R, \mu}=H_{\lambda}^{R, \mu}+C_{\lambda}$, which gives an identification

$$
S_{n}^{[\eta, b[}\left(K_{\lambda}^{R, \mu}\right)=S_{n}^{\left[\eta+C_{\lambda}, b+C_{\lambda}[\right.}\left(H_{\lambda}^{R, \mu}\right) .
$$

Using a result in [7], we can identify $J_{\lambda}^{b} \circ I_{\lambda}^{b}$ with the natural map

$$
S_{n}^{[\eta, b[}\left(H_{\lambda}^{R, \mu}\right) \longrightarrow S_{n}^{\left[\eta+C_{\lambda}, b+C_{\lambda}[\right.}\left(H_{\lambda}^{R, \mu}\right) .
$$

This map vanishes as soon as $C_{\lambda}+\eta>b$, which is true if $\lambda$ is large enough. We just proved that $J_{\lambda}^{b}\left(x_{0}\right)=0$ for large enough $\lambda$. Consider now the Hamiltonian functions $F_{\mu}=F_{0}^{R, \mu}+\eta$ and $K_{\lambda}=K_{\lambda}^{R, \mu}+\eta$, and notice that $K_{\lambda}$ is still a cofinal family in $\mathcal{H}_{\mathrm{FH}}(V)$. As before, we can identify $J_{\lambda}^{b}$ with the monotonicity map

$$
j_{\lambda}^{b-\eta}: \mathbb{Z}_{2} \cdot x_{0}=S_{n}^{[0, b-\eta[}\left(F_{\mu}\right) \longrightarrow S_{n}^{[0, b-\eta[}\left(K_{\lambda}\right),
$$

and we know that if $b=c_{\mathrm{V}}(U)$, then $j_{\lambda}^{b}\left(x_{0}\right)=0$ for large enough $\lambda$. On the other hand, set $f=-3 \delta+\nu \tau_{0}$ for small enough $\nu>0$, and consider the commutative diagram

$$
\begin{array}{r}
S_{n}^{[0, \varepsilon[}(f)=\mathbb{Z}_{2} \cdot x_{0} \stackrel{i_{f}}{\longrightarrow} S_{n}^{[0, \varepsilon[}\left(F_{\mu}\right)=\mathbb{Z}_{2} \cdot x_{0} \stackrel{j_{\lambda}^{\varepsilon}}{\longrightarrow} S_{n}^{[0, \varepsilon[}\left(K_{\lambda}\right) \\
i_{0}^{b} \downarrow \\
S_{n}^{[0, b[}\left(F_{\mu}\right)=\mathbb{Z}_{2} \cdot x_{0} \stackrel{j_{\lambda}^{b}}{\longrightarrow} S_{n}^{[0, b[}\left(K_{\lambda}\right)
\end{array}
$$

where $3 \delta<\varepsilon \leqslant \pi r$ : we have $\sigma_{f}=j_{\lambda}^{\varepsilon} \circ i_{f}$. As in Proposition 3.3, we see that $i_{f}\left(x_{0}\right)=x_{0}$, and that $j_{\lambda}^{\varepsilon}\left(x_{0}\right)=\sigma_{f}\left(x_{0}\right)=\alpha_{\lambda}$ is nonzero. Moreover, we see that $i_{\lambda}^{b}\left(\alpha_{\lambda}\right)=j_{\lambda}^{b}\left(i_{0}^{b}\left(x_{0}\right)\right)=0$, which implies $b \geqslant c_{\mathrm{FH}}\left(K_{\lambda}\right)$. Applying Proposition 3.3, we infer:

$$
c_{\mathrm{FH}}(V)=\lim _{\lambda \rightarrow+\infty} c_{\mathrm{FH}}\left(K_{\lambda}\right) \leqslant b=c_{\mathrm{V}}(U),
$$

which finishes the proof of Theorem 1.4.

5.2. The case of RCT open sets. - Let $U$ be a RCT open set: we know that $c_{\mathrm{FH}}(U) \leqslant c_{\mathrm{V}}(U)$. We will use the intrinsic description of the capacity $c_{\mathrm{FH}}$ given in [7] in order to prove the opposite inequality. In view of (1.5), it suffices to deal with the case where $\partial U$ has a nondegenerate action spectrum, because this property is $C^{\infty}$-generic among hypersurfaces. We proved in [7] that we have an isomorphism

$$
S_{k}^{[a, b[}(U) \stackrel{\sim}{\longrightarrow} I S_{k}^{[a, b[}(\bar{U}) \text { for any } k \in \mathbb{Z},-\infty<a \leqslant b \leqslant+\infty .
$$

Let us recall how this isomorphism is defined. The map $\Psi$ given by (3.1) defines an exact symplectic embedding of $\widehat{U}$ into $\mathbb{C}^{n}$. Let $J_{U}$ be the special almost complex structure on $\widehat{\partial U}$ defined in Section 3.1, and let $A, B$ be real numbers such that $B>2 A>2$. Let $J$ be a calibrated almost complex structure on $\mathbb{C}^{n}$

TOME $132-2004-\mathrm{N}^{\mathrm{O}} 4$ 


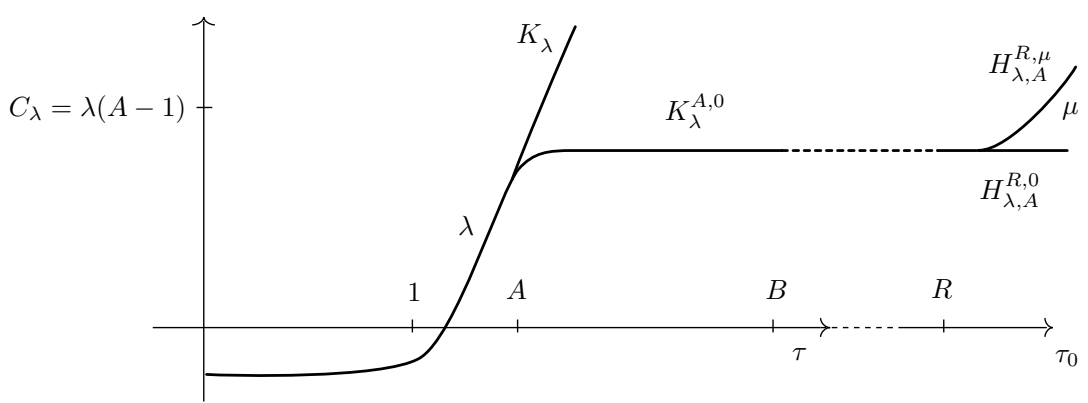

FiguRE 5. "Intrinsic functions" $K_{\lambda}^{A, 0} \prec K_{\lambda}$ and "relative functions" $H_{\lambda, A}^{R, 0} \prec H_{\lambda, A}^{R, \mu}$

satisfying $J=\Psi_{*} J_{U}$ in $U^{B} \backslash U^{A}$ and $J=i$ at infinity. On the other hand, consider the cofinal family $K_{\lambda}: \widehat{U} \rightarrow \mathbb{R}$ in (3.6), and deform $K_{\lambda}$ into $K_{\lambda}^{A, 0}: \widehat{U} \rightarrow \mathbb{R}$ by setting

$$
K_{\lambda}^{A, 0}= \begin{cases}K_{\lambda} & \text { in } U^{A} \\ C_{\lambda} & \text { off } U^{A}\end{cases}
$$

where $C_{\lambda}=\lambda(A-1)$. Extend the function $H_{\lambda, A}^{R, 0}=\Psi_{*} K_{\lambda}^{A, 0}$ to $\mathbb{C}^{n}$ by $H_{\lambda, A}^{R, 0}=C_{\lambda}$ of $U^{A}$. Finally, choose a real number $R>B R_{0}$, which implies $U^{B} \subset B^{R}$, and deform $H_{\lambda, A}^{R, 0}$ into $H_{\lambda, A}^{R, \mu}$ by setting

$$
H_{\lambda, A}^{R, \mu}= \begin{cases}H_{\lambda, A}^{R, 0} & \text { inside } B^{R} \\ C_{\lambda}+\mu\left(\tau_{0}-R\right) & \text { off } B^{R}\end{cases}
$$

(see Figure 5). Then perform the same perturbations as in Section 3.1, that is, smooth the corners, introduce a $C^{2}$-small Morse perturbation inside $U$, then a time-dependent perturbation of both $H_{\lambda, A}^{R, \mu}$ and $J$ near $\bar{U}$. Given $a, b \in \mathbb{R}$ such that $a<b$, we can choose the parameters so that

- $H_{\lambda, A}^{R, \mu}$ is a cofinal family in $\mathcal{H}_{\mathrm{QI}}(U)$ and $S_{k}^{[a, b[}\left(H_{\lambda, A}^{R, \mu}\right)$ is well defined

- all orbits of $H_{\lambda, A}^{R, \mu}$ with action in [a,b stay in $U_{1+\nu}$ and all Floer trajectories connecting them stay in $U^{A}$ (see below).

Thus we can identify $S_{k}^{[a, b[}\left(H_{\lambda, A}^{R, \mu}\right)$ with $S_{k}^{[a, b[}\left(K_{\lambda}\right)$ and get the isomorphism in (5.1). In the language of [16], if $U \subset V$ are RCT open sets, the resulting map

$$
I S_{k}^{[a, b[}(\bar{V})=S_{k}^{[a, b[}(V) \longrightarrow S_{k}^{[a, b[}(U)=I S_{k}^{[a, b[}(\bar{U})
$$

is the transfer morphism. In particular, if $\varepsilon$ is small enough, then $S_{n}^{[0, \varepsilon[}(U)$ is one-dimensional in view of (3.8). As a result, the class $\alpha_{U}$ in Section 3.2 is its generator $x_{0}$, and we have

$$
c_{\mathrm{FH}}(U)=\inf \left\{b / i_{\widehat{U}}^{b}\left(x_{0}\right)=0\right\},
$$

BULLETIN DE LA SOCiÉTÉ MATHÉMATIQUe DE FRANCE 


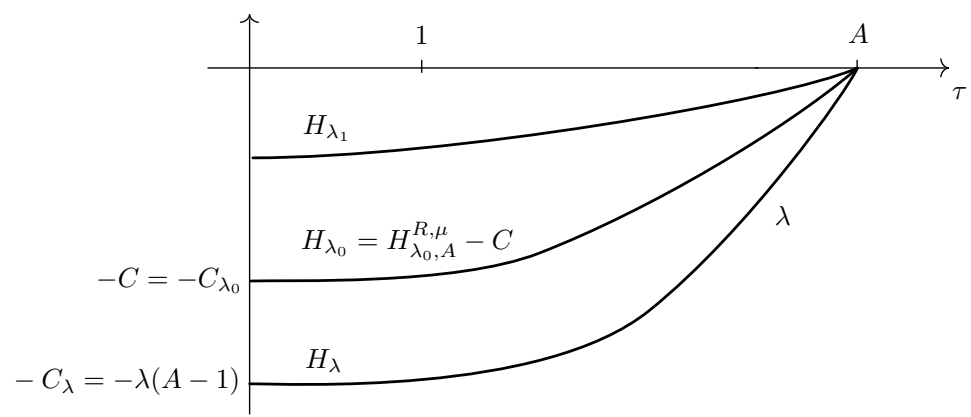

FIGURE 6. Compactly supported cofinal family $H_{\lambda}$ where $\lambda_{1} \ll \lambda_{0}<\lambda$

where $i_{\widehat{U}}^{b}: I S_{n}^{[0, \varepsilon[}(\bar{U}) \rightarrow I S_{n}^{[0, b[}(\bar{U})$ is the natural map. Moreover, Property (3.6) allows us to characterize $c_{\mathrm{FH}}(U)$ in the following way: choose any real numbers $\varepsilon$ and $b$ such that $\delta<\varepsilon<T_{0} \leqslant c_{\mathrm{FH}}(U)<b$, where $T_{0}$ is the least period of a closed characteristic of $\partial U$. If $\lambda_{0}>b$ and $\lambda_{0} \notin \mathcal{S}(\partial U)$, we infer that the map

$$
\mathbb{Z}_{2} \cdot x_{0}=S_{n}^{[0, \varepsilon[}\left(K_{\lambda_{0}}\right) \longrightarrow S_{n}^{[0, b[}\left(K_{\lambda_{0}}\right)
$$

sends the class of $x_{0}$ to zero. In other terms, we see $c_{\mathrm{FH}}(U)$ as the first slope where $c_{\mathrm{FH}}\left(K_{\lambda}\right)$ is finite. Now consider the function $K_{\lambda_{0}}^{A, 0}$ defined above, whose only degenerate orbits are the constants off $U^{A}$, at level $-C=-C_{\lambda_{0}}$. Since $\mathcal{S}(\partial U)$ is nondegenerate, there exists a real number $\eta_{0}>0$ such that $\mathcal{S}(\partial U) \cap\left[\lambda_{0}-\eta_{0}, \lambda_{0}\right]=\varnothing$. This implies that under the deformation

$$
K_{\lambda_{0}}^{A, 0} \rightsquigarrow K_{\lambda_{0}}^{A, \lambda_{0}}=K_{\lambda_{0}}
$$

new 1-periodic orbits can appear only below the level $-\lambda_{0}(A-1)+\left(\lambda_{0}-\eta_{0}\right) A=$ $\lambda_{0}-\eta_{0} A$, which is negative for large enough $A$. This shows that the monotonicity morphism

$$
S_{k}^{[a, b[}\left(K_{\lambda_{0}}^{A, 0}\right) \longrightarrow S_{k}^{[a, b[}\left(K_{\lambda_{0}}\right) \text { is an isomorphism if } a>\lambda_{0}-\eta_{0} A .
$$

Moreover, since $J=\Psi_{*} J_{U}$ in $U^{B} \backslash U^{A}$, if a Floer trajectory associated to $J$ and $H_{\lambda_{0}, A}^{R, 0}$ exits $U^{A}$, it must exit $U^{B}$ in view of the maximum principle, and its energy is at least $(B-A) C\left(J_{U}\right)$ in view of (3.3), which is impossible for large enough $B$. Thus we have

$$
S_{k}^{[a, b[}\left(H_{\lambda_{0}, A}^{R, 0}\right)=S_{k}^{[a, b[}\left(K_{\lambda_{0}}^{A, 0}\right) \text { if } a>-C .
$$

This shows that if $A$ is large enough, the natural map

$$
\mathbb{Z}_{2} \cdot x_{0}=S_{n}^{[0, \varepsilon[}\left(H_{\lambda_{0}, A}^{R, 0}\right) \longrightarrow S_{n}^{[0, b[}\left(H_{\lambda_{0}, A}^{R, 0}\right)
$$

sends $x_{0}$ to zero. Now consider the function $H_{\lambda_{0}}=H_{\lambda_{0}, A}^{R, 0}-C$ : it has support in $S^{1} \times U^{A}$, and we just proved that the natural map

TOME $132-2004-\mathrm{N}^{\mathrm{O}} 4$ 


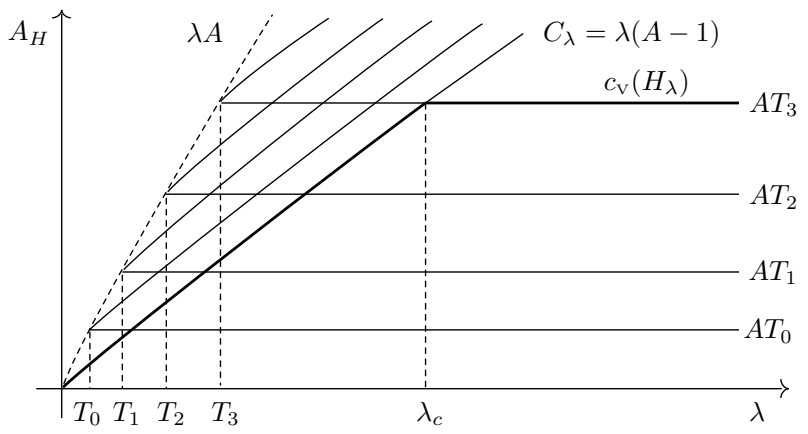

FiguRE 7 . Bifurcation diagram of the family $H_{\lambda}$

$$
j_{\lambda_{0}}^{C+b}: \mathbb{Z}_{2} \cdot x_{0}=S_{n}^{[C, C+\varepsilon[}\left(H_{\lambda_{0}}\right) \rightarrow S_{n}^{[C, C+b[}\left(H_{\lambda_{0}}\right) \text { fulfills } j_{\lambda_{0}}^{C+b}\left(x_{0}\right)=0 .
$$

This shows that $x_{0}$ is not the element $\gamma_{\lambda_{0}}$ in Proposition 4.5, because otherwise we would have $j_{\lambda_{0}}^{C+b}\left(x_{0}\right) \neq 0$. On the other hand, define a cofinal family in $\mathcal{H}_{\mathrm{CS}}\left(U^{A}\right)$ as follows (see Figure 6 ). Given any real number $\lambda>0$, set

$$
H_{\lambda}= \begin{cases}\lambda(1-A) & \text { in } U \\ \lambda(\tau-A) & \text { in } U^{A} \backslash U, \\ 0 & \text { off } U^{A} .\end{cases}
$$

After the same perturbations as above, we get a cofinal family $H_{\lambda} \in \mathcal{H}_{\mathrm{V}}\left(U^{A}\right)$, equal to the above $H_{\lambda_{0}}$ for $\lambda=\lambda_{0}$. Its 1-periodic orbits are (see Figure 7):

- constants in $U$ with action near $C_{\lambda}=\lambda(A-1)$ and constants off $U^{A}$ with zero action;

- periodic orbits near $\partial U^{A}$ with action near $T_{k} A$, where $T_{k} \in \mathcal{S}(\partial U)$ and $T_{k} \leqslant \lambda$;

- periodic orbits near $\partial U$ with action near $C_{\lambda}+T_{k}$, where $T_{k}$ is as above.

We know that $c_{\mathrm{V}}\left(H_{\lambda}\right)=A_{H_{\lambda}}\left(\gamma_{\lambda}\right)$ is a critical value of $A_{H_{\lambda}}$, that it is continuous with respect to $\lambda$, and that it is bounded above by $c_{\mathrm{V}}\left(U^{A}\right)$. We also know that if $\lambda$ is small enough, then $c_{\mathrm{V}}\left(H_{\lambda}\right)=-\min H_{\lambda}$. This implies that there exists a slope $\lambda_{\mathrm{c}}$ such that

- if $\lambda<\lambda_{\mathrm{c}}$ then $\gamma_{\lambda}$ is the constant orbit $x_{0}$

- if $\lambda>\lambda_{c}$ then $\gamma_{\lambda}$ is a periodic orbit near $\partial U^{A}$ with action near $T_{k} A$, for some fixed $T_{k} \in \mathcal{S}(\partial U)$ satisfying $T_{k}<\lambda_{\mathrm{c}}$.

Since $H_{\lambda}$ is a cofinal family in $\mathcal{H}_{\mathrm{V}}\left(U^{A}\right)$, we get $c_{\mathrm{V}}\left(U^{A}\right)=T_{k} A$, which implies that we have $c_{\mathrm{V}}(U)=T_{k}<\lambda_{\mathrm{c}}$. Thus we have seen $c_{\mathrm{V}}(U)$ as the first bifurcation slope for $c_{\mathrm{V}}\left(H_{\lambda}\right)$. Since $\gamma_{\lambda_{0}}$ is not the constant $x_{0}$ (see above), we have 
necessarily $\lambda_{0} \geqslant \lambda_{\mathrm{c}}$, thus $\lambda_{0}>c_{\mathrm{V}}(U)$. Since this holds for any $\lambda_{0}>c_{\mathrm{FH}}(U)$, we infer $c_{\mathrm{FH}}(U) \geqslant c_{\mathrm{V}}(U)$, which is the desired inequality.

5.3. The Lagrangian camel. - Let $L \subset \mathbb{C}^{n}$ be any compact Lagrangian submanifold and choose any (time-independent) almost complex structure $J \in \mathcal{J}$. We will now prove Theorem 1.6, that is, there exists some $J$-holomorphic curve $C$ with boundary in $L$ satisfying

$$
\int_{C} \omega \leqslant c_{F H}(L)
$$

This theorem is contained in [16] (see Theorem 6.10), but its proof can not be translated verbatim to our language, so we write it down for the convenience of the reader: the rescaling process does not allow us to construct suitable cofinal families (see below), and we must take into account non-contractible orbits. Consider the cotangent bundle $T^{*} L$, with canonical symplectic form $\omega=\mathrm{d} \lambda$, where $\lambda=p \mathrm{~d} q$. Fix a Riemannian metric on $L$ and endow $T^{*} L$ with the associated metric: the unit disk bundle

$$
D=\left\{(q, p) \in T^{*} L /|p| \leqslant 1\right\}
$$

has RCT boundary, and we have $\widehat{D}=T^{*} L$ and $\tau(q, p)=|p|^{2}$. The action spectrum $\mathcal{S}$ of $D$ is the set of lengths of closed geodesics on $L$, and it is nondegenerate if the metric on $L$ is generic (the Conley-Zehnder index of the periodic orbit depends on the Morse index of the closed geodesic, see [13]). Remember from (3.8) in Section 3.1 that for small enough $\varepsilon$ we have

$$
I S_{k}^{[0, \varepsilon[}(D)=F H_{k}^{[0, \varepsilon[}(D)=H_{2 n-k}(D, \partial D)=H^{k}(L) .
$$

The crucial fact, which is proved in [15], is the following theorem.

Theorem 5.1. - For any $b \geqslant \varepsilon$, the natural map $j_{D}^{b}: I S_{k}^{[0, \varepsilon[}(D) \rightarrow I S_{k}^{[0, b[}(D)$ is injective.

Indeed, $I S_{*}^{[0,+\infty[}(D)$ is isomorphic to the homology of the free loop space on $L$, and the natural map $j_{D}^{\infty}$ is induced by the inclusion of constant loops.

According to Weinstein's Theorem, we have a symplectic embedding

$$
\Psi: D^{\sigma} \longrightarrow \mathbb{C}^{n}
$$

for some $\sigma>0$. By homogeneity, we can rescale the metrics so that $\sigma=3$ and $\Psi(D)$ contains a unit ball $B$ centered on $L$, and we fix $R>0$ satisfying $\Psi\left(D^{3}\right) \subset B^{R}$. The main point is the following: Theorem 5.1 and the definition of $c_{\mathrm{FH}}(L)$ imply that if $b>c_{\mathrm{FH}}(L)$ and if $\sigma$ is small enough, then $S_{n}^{[0, b[}\left(\Psi\left(D^{\sigma}\right)\right)$ is not isomorphic to $I S_{n}^{[0, b[}\left(D^{\sigma}\right)$. For a well chosen family of Hamiltonian functions, this will imply the existence of a family of Floer trajectories which converges as a family of current to a $J$-holomorphic curve with boundary in $L$. Since $L$ is not exact, $\Psi(D)$ is not a RCT open set in $\mathbb{C}^{n}$ and we cannot make the

TOME $132-2004-\mathrm{N}^{\mathrm{O}} 4$ 


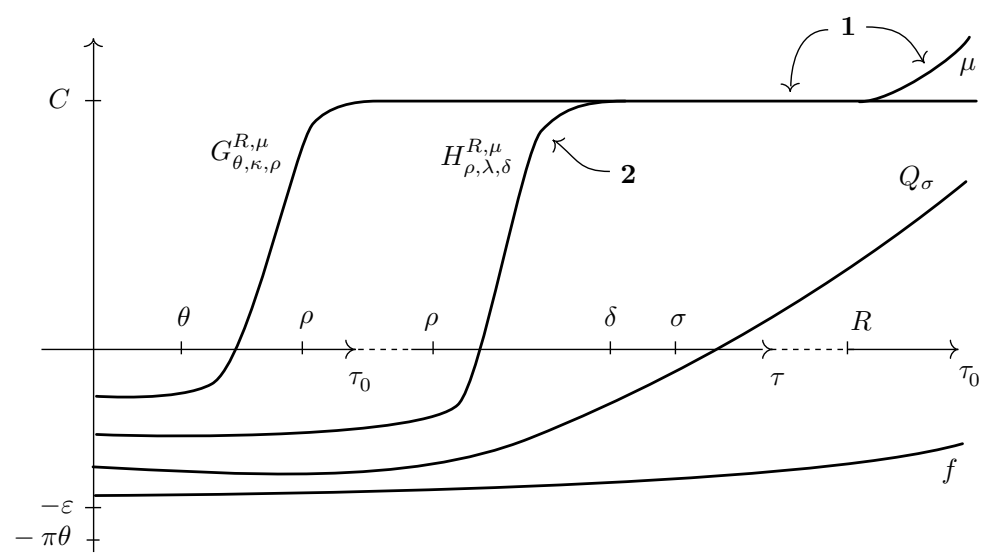

FIgURE 8. Functions $H=H_{\rho, \lambda, \delta}^{R, \mu} \prec G_{\theta, \kappa, \rho}^{R, \mu}=G$ and $H^{\delta}=H_{\rho, \lambda, \delta}^{R, 0} \prec G_{\theta, \kappa, \rho}^{R, 0}=G^{\rho}$

same constructions as in Section 5.2 for two reasons. The first one is that $\Psi$ does not extend to the whole $T^{*} L$, thus we must rescale the Hamiltonian functions of Section 5.2. The other problem, which explains the positivity of $c_{\mathrm{FH}}(L)$, is that $\Psi_{*} \lambda$ does not extend to $\mathbb{C}^{n}$ as a primitive of $\omega$. As a consequence, the canonical action in $T^{*} L$ equals the action in $\mathbb{C}^{n}$ only for those orbits which are contractible in $D$, and we must take care of the splitting

$$
I S_{k}^{[a, b[}(D)=F H_{k}^{[a, b[}(D) \oplus N C_{k}^{[a, b[}(D) .
$$

Start with any real number $0<\delta \leqslant 1$, and choose a (time-independent) almost complex structure $J_{1} \in \mathcal{J}$ satisfying

$$
J_{1}=\Psi_{*} J_{D} \text { in } \Psi\left(D^{2 \delta} \backslash D^{\delta}\right) \quad \text { and } \quad J_{1}=J \text { off } \Psi\left(D^{3 \delta}\right),
$$

where $J_{D}$ is the special almost complex structure on $\widehat{\partial D}$ defined in Section 3.1. Given real numbers $\rho$ and $\lambda$ satisfying $0<\rho<\delta, \lambda>0$ and $\lambda \notin \mathcal{S}$, consider the functions given by

$K_{\rho, \lambda}=\left\{\begin{array}{ll}0 & \text { in } D^{\rho}, \\ \lambda(\tau-\rho) & \text { off } D^{\rho},\end{array} \quad K_{\rho, \lambda}^{\delta, 0}=\left\{\begin{array}{ll}K_{\rho, \lambda} & \text { in } D^{\delta}, \\ C & \text { off } D^{\delta},\end{array} \quad\right.\right.$ where $C=\lambda(\delta-\rho)$.

Extend the function $H_{\rho, \lambda, \delta}^{R, 0}=\Psi_{*} K_{\rho, \lambda}^{\delta, 0}$ to $\mathbb{C}^{n}$, then deform $H_{\rho, \lambda, \delta}^{R, 0}$ into $H=H_{\rho, \lambda, \delta}^{R, \mu}$, where

$$
H_{\rho, \lambda, \delta}^{R, \mu}= \begin{cases}H_{\rho, \lambda, \delta}^{R, 0} & \text { inside } B^{R} \\ C+\mu\left(\tau_{0}-R\right) & \text { off } B^{R}\end{cases}
$$

(see Figure 8). Perform the same perturbations as above, that is, smooth the corners, replace the constant 0 by a small Morse function, and introduce a 
time-dependent perturbation of both $H$ and $J_{1}$ in a neighborhood of the orbits with positive action. Let $\eta>0$ be a real number satisfying $[\lambda-\eta, \lambda] \cap \mathcal{S}=\varnothing$ : aside the orbits of $K_{\rho, \lambda}$, the Hamiltonian function $H$ has the following orbits

- constants in $B^{R} \backslash D^{\delta}$ and orbits near $\partial B^{R}$ : we call them orbits of type $\mathbf{1}$, and their action is at most $-C+\mu R$, where $C=\lambda(\delta-\rho)$;

- orbits near $\partial D^{\delta}$, which we call orbits of type $\mathbf{2}$. Let orbits of type $\mathbf{2}^{\mathbf{c}}$ denote those orbits of type $\mathbf{2}$ which are contractible in $D$ : their action is at most $\lambda \rho-\eta \delta$.

Now we will choose the parameters: choose any real number $\mu>\pi$, and assume that $\rho<\frac{1}{2} \delta$. Then the orbits of type 1 have action at most $\mu R-\frac{1}{2} \delta \lambda$. Choose any number $\lambda>2 \mu R / \delta$ such that $\lambda \notin \mathcal{S}$, which ensures that all these orbits have negative action. Next, we get a number $\eta>0$ satisfying $[\lambda-\eta, \lambda] \cap \mathcal{S}=\varnothing$, and we choose any real number $\rho$ such that $0<\rho<\eta \delta / \lambda$ : this ensures that the orbits of type $\mathbf{2}^{\mathbf{c}}$ have negative action. Observe that the only parameters we are free to choose are $\delta$ and $\mu$ : since we require $\lambda \rho<\eta \delta$, we do not get a cofinal family in $\mathcal{H}_{\mathrm{QI}}\left(\Psi\left(D^{\rho}\right)\right)$ for a given $\rho>0$. However, we know that $H \in \mathcal{H}_{\mathrm{FH}}^{\mathrm{c}}$ (see Section 3.2), and we have $H \geqslant C>\mu R$ off $D^{\delta}$, where $R$ is fixed. Let $b$ be any number such that

$$
b>c_{\mathrm{FH}}(L)=\lim _{\sigma \rightarrow 0} c_{\mathrm{FH}}\left(\Psi\left(D^{\sigma}\right)\right)
$$

and let $\sigma$ be a real number such that $c_{\mathrm{FH}}\left(\Psi\left(D^{\sigma}\right)\right)<b$. By definition, there exists some $Q^{\sigma} \in \mathcal{H}_{\mathrm{FH}}^{\mathrm{c}}\left(\Psi\left(D^{\sigma}\right)\right)$ satisfying $c_{\mathrm{FH}}\left(Q^{\sigma}\right)<b$. If we have $\delta<\sigma$ and if $\mu$ is large enough, then we have $H \geqslant Q^{\sigma}$ on $S^{1} \times \mathbb{C}^{n}$, and Proposition 3.3 implies that $c_{\mathrm{FH}}(H) \leqslant c_{\mathrm{FH}}\left(Q^{\sigma}\right)$. We have just proved that

$$
c_{\mathrm{FH}}(H)=c_{\mathrm{FH}}\left(H_{\rho, \lambda, \delta}^{R, \mu}\right)<b \quad \text { for small enough } \delta \text { and large enough } \mu .
$$

In order to use this result, we will now build a family of Hamiltonian functions representing a small ball as in Section 3.2. Observe that we have $B^{\rho} \subset D^{\rho}$. Given real numbers $\theta$ and $\kappa$ such that $0<\theta<\rho, \kappa>0$ and $\kappa \notin \pi \mathbb{Z}$, consider the functions given by

$$
F_{\theta, \kappa}=\left\{\begin{array}{ll}
0 & \text { in } B^{\theta}, \\
\kappa\left(\tau_{0}-\theta\right) & \text { off } B^{\theta},
\end{array} \quad F_{\theta, \kappa}^{\rho, 0}= \begin{cases}F_{\theta, \kappa} & \text { in } B^{\rho}, \\
\kappa(\rho-\theta) & \text { off } B^{\rho}\end{cases}\right.
$$

and deform $G_{\theta, \kappa, \rho}^{R, 0}=F_{\theta, \kappa}^{\rho, 0}$ into $G=G_{\theta, \kappa, \rho}^{R, \mu}$ as above. Perform the same perturbations as before: we can choose the parameters so that $\theta>\frac{1}{2} \rho$ and $\kappa(\rho-\theta)=C$, which implies

$$
G_{\theta, \kappa, \rho}^{R, \mu} \geqslant H_{\rho, \lambda, \delta}^{R, \mu} \text { on } S^{1} \times \mathbb{C}^{n} \quad \text { and } \quad G_{\theta, \kappa, \rho}^{R, \mu}=H_{\rho, \lambda, \delta}^{R, \mu} \text { off } D^{\delta} .
$$

As in Proposition 3.3, consider the function $f=\nu \tau_{0}-\beta$ where $\beta>0$ and $\nu>0$ are small enough, so that $f \leqslant H$ on $S^{1} \times \mathbb{C}^{n}$. If $\beta<\varepsilon<\pi \theta$, we have a

TOME $132-2004-\mathrm{N}^{\mathrm{O}} 4$ 
commutative diagram

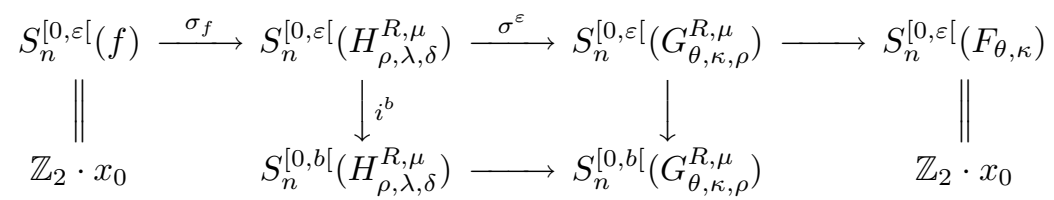

where $x_{0}$ is the center of the ball, and $\alpha=\sigma_{f}\left(x_{0}\right)$ satisfies $i^{b}(\alpha)=0$ in view of (5.4). Since the map $S_{n}^{[0, \varepsilon[}(f) \rightarrow S_{n}^{[0, \varepsilon[}\left(F_{\theta, \kappa}\right)$ is an isomorphism, we infer $\sigma^{\varepsilon}(\alpha) \neq 0$. Moreover, for any real number $c>0$ we have a commutative diagram

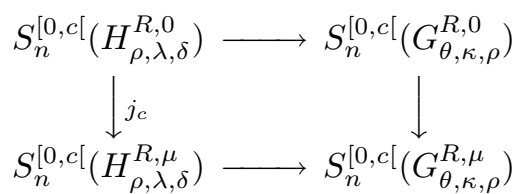

where the vertical arrows are isomorphisms. Indeed, during the deformation $0 \rightsquigarrow \mu$, the only orbits that appear have type $\mathbf{1}$, and they have negative action. Set $\alpha_{0}=j_{\varepsilon}^{-1}(\alpha)$ : the commutative diagram

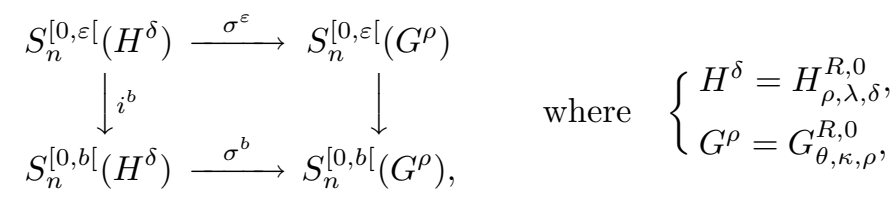

shows that we have $i^{b}\left(\alpha_{0}\right)=0$ and $\sigma^{\varepsilon}\left(\alpha_{0}\right) \neq 0$. On the other hand, consider the natural map

$$
j_{D}^{b}: \mathbb{Z}_{2} \cdot x_{0}=S_{n}^{[0, \varepsilon[}\left(K_{\rho, \lambda}\right) \longrightarrow S_{n}^{[0, b[}\left(K_{\rho, \lambda}\right),
$$

where $x_{0}$ is the minimum of $K_{\rho, \lambda}$. In view of Theorem 5.1 and (3.6), we have $j_{D}^{b}\left(x_{0}\right) \neq 0$. As above, since orbits of type $\mathbf{2}^{\mathbf{c}}$ have negative action, we infer that the map

$$
i_{D}^{b}: \mathbb{Z}_{2} \cdot x_{0}=S_{n}^{[0, \varepsilon[}\left(K^{\delta}\right) \longrightarrow S_{n}^{[0, b[}\left(K^{\delta}\right), \quad \text { where } \quad K^{\delta}=K_{\rho, \lambda}^{\delta, 0}
$$

fulfills $i_{D}^{b}\left(x_{0}\right) \neq 0$. Since $x_{0}$ is the only orbit of $H^{\delta}$ with action near 0 which is contractible in $D$, we have two possibilities for arbitrarily small $\delta>0$ : either $\alpha_{0}$ is not contractible in $D$, or $\alpha_{0}=x_{0}$. In the first case, since $\sigma^{\varepsilon}\left(\alpha_{0}\right) \neq 0$ we have a monotonicity trajectory connecting $\alpha_{0}$ to an orbit of $G^{\rho}$ : this orbit is not of type $\mathbf{1}$, because it has positive action, thus it is contractible in $B^{\rho} \subset D^{\rho}$. Since $\alpha_{0}$ is not contractible, the monotonicity trajectory must exit $D$. Since we have $G^{\rho}=H^{\delta}$ and $J_{1}=\Psi_{*} J_{D}$ in $\psi\left(D^{2 \delta} \backslash D^{\delta}\right)$, the corresponding part of this trajectory is a holomorphic curve with area at most $\varepsilon$ and at least $\delta C\left(J_{D}\right)$. This is impossible for small enough $\delta$, because we have $\varepsilon<\pi \theta$ and $\theta$ is much smaller than $\delta$.

BULLETIN DE LA SOCIÉtÉ MATHÉMATIQUE DE FRANCE 
Thus we have $\alpha_{0}=x_{0}$ and we can use the arguments in [16]: since $i^{b}\left(x_{0}\right)=0$, there exists an odd number of Floer trajectories for $H^{\delta}$ which connect $x_{0}$ to orbits with action less than $b$. One of these trajectories must exit $D^{3 \delta}$, because otherwise they would be Floer trajectories for $K^{\delta}$, which would contradict $i_{D}^{b}\left(x_{0}\right) \neq 0$. Let $u$ be the part of this trajectory located off $D^{3 \delta}$. Since $H^{\delta}$ is constant and $J_{1}=J$ off $D^{3 \delta}$, the map $u$ is a $J$-holomorphic curve with area at most $b$. When $\delta$ goes to zero, the $J$-holomorphic curve $u$ converges as a current to a $J$-holomorphic curve with boundary in $L$ and with area at most $b$. This shows that $\widetilde{w}(L) \leqslant b$. Since this holds for any $b>c_{\mathrm{FH}}(L)$, we have proved Theorem 1.6.

\section{Some open questions}

The main idea in the search for comparisons of symplectic capacities is that the various known symplectic capacities have different properties, which makes the interest of their classification. This is illustrated by Corollary 1.7: the Floer-Hofer capacity is nonzero for compact Lagrangian submanifolds, and the Viterbo capacity is an obstruction to the camel problem, thus we solved the Lagrangian camel problem by proving the inequality $c_{\mathrm{V}}(L) \geqslant c_{\mathrm{FH}}(L)$. More generally, in a manifold with RCT boundary which satisfies the strong algebraic Weinstein conjecture, we have on one hand outer regular (QI)-capacities defined by symplectic homology as in Section 3, and on the other hand inner regular (CS)-capacities defined by symplectic homology with compact support (see (4.6) and Remark 4.2). Even for regular domains, the opposite regularity of these Hamiltonian capacities sounds very hard to prove, and might be wrong. Notice that the method used in Section 5.1 can only show that (CS)-capacities are larger than (QI)-capacities, since $J_{\lambda}^{b} \circ I_{\lambda}^{b}$ finally vanishes for any $b$, and we do not know whether we have $\check{c}_{\mathrm{FH}}=c_{\mathrm{V}}$ in general. However, it is more reasonable to compare capacities when they have a geometric representation and interesting applications, that is, for open sets with contact type boundary. The common guess is that Hamiltonian capacities are all equal in this case, but we do not know whether we have $c_{\mathrm{FH}}(L)=c_{\mathrm{V}}(L)$ for any compact Lagrangian submanifold $L \subset \mathbb{C}^{n}$.

\section{BIBLIOGRAPHY}

[1] Cieliebak (K.), Floer (A.) \& Hofer (H.) - Symplectic homology II (a general construction), Math. Z., t. 218 (1995), pp. 103-122.

[2] Cieliebak (K.), Floer (A.), Hofer (H.) \& Wysocky (K.) - Applications of symplectic homology II (stability of the action spectrum), Math. Z., t. 223 (1996), pp. 27-45.

TOME $132-2004-\mathrm{N}^{\mathrm{O}} 4$ 
[3] Ekeland (I.) \& Hofer (H.) - Symplectic topology and Hamiltonian dynamics, Math. Z., t. 200 (1990), pp. 355-378.

[4] Floer (A.) \& Hofer (H.) - Symplectic homology I (open sets in $\mathbb{C}^{n}$ ), Math. Z., t. 215 (1994), pp. 37-88.

[5] Floer (A.), Hofer (H.) \& Wysocki (K.) - Applications of symplectic homology I, Math. Z., t. 217 (1994), pp. 577-606.

[6] Gromov (M.) - Pseudo holomorphic curves in symplectic manifolds, Invent. Math., t. 82 (1985), pp. 307-347.

[7] Hermann (D.) - Holomorphic curves and Hamiltonian systems in an open set with restricted contact type boundary, Duke Math. J., t. 103 (2000), no. 2, pp. 335-374.

[8] Hofer (H.) \& ZeHNDER (E.) - Symplectic invariants and Hamiltonian dynamics, Birkhäuser, 1994.

[9] Salamon (D.) \& Zehnder (E.) - Morse theory for periodic solutions of Hamiltonian systems and the Maslov index, Comm. Pure Appl. Math., t. 45 (1992), pp. 1303-1360.

[10] Théret (D.) - A Lagrangian camel, Comment. Math. Helv., t. 74 (1999), no. 4 , pp. $591-614$.

[11] _ A complete proof of Viterbo's uniqueness theorem on generating functions, Topology Appl., t. 96 (1999), no. 3, pp. 246-266.

[12] Traynor (L.) - Symplectic homology via generating functions, Geom. Funct. Anal., t. 4 (1994), no. 6, pp. 718-748.

[13] Viterbo (C.) - A new obstruction to embedding Lagrangian tori, Invent. Math., t. 100 (1990), pp. 301-320.

[14] Symplectic topology as the geometry of generating functions, Math. Ann., t. 292 (1992), pp. 685-710.

[15] - Functors and computations in Floer homology with applications II, (1998), Preprint Université Paris-Sud 98-15. To appear in GAFA.

[16] Functors and computations in Floer homology with applications I, Geom. Funct. Anal., t. 9 (1999), no. 5, pp. 985-1033. 